\title{
Handover Performance over a Coordinated Contiguous Carrier Aggregation Deployment Scenario in the LTE-Advanced System
}

\author{
Ibraheem Shayea, ${ }^{1}$ Mahamod Ismail, ${ }^{1}$ Rosdiadee Nordin, ${ }^{1}$ and Hafizal Mohamad ${ }^{2}$ \\ ${ }^{1}$ Department of Electronics, Electrical and System Engineering, Faculty of Engineering and Build Environment, \\ Universiti Kebangsaan Malaysia, 43600 Bangi, Selangor, Malaysia \\ ${ }^{2}$ MIMOS Berhad, Technology Park Malaysia, 57000 Kuala Lumpur, Malaysia
}

Correspondence should be addressed to Ibraheem Shayea; shaia2009@yahoo.com

Received 25 May 2014; Revised 26 July 2014; Accepted 27 July 2014; Published 31 August 2014

Academic Editor: Martin Reisslein

\begin{abstract}
Copyright (C) 2014 Ibraheem Shayea et al. This is an open access article distributed under the Creative Commons Attribution License, which permits unrestricted use, distribution, and reproduction in any medium, provided the original work is properly cited.

Although various carrier aggregation deployment scenarios (CADSs) have been introduced in the LTE-Advanced system, issues related to insufficient eNB coverage that leads to low throughput and high drop call have yet to be solved. This paper proposes a new deployment scenario called coordinated contiguous-CADS (CC-CADS), which utilizes two-component carriers (CCs) that operate on two frequencies located in a contiguous band. Each CC antenna is directed to a cell boundary of the other CC. The handover performance of users with various mobility speeds under CC-CADS has been investigated and compared with various deployment scenarios proposed by 3GPP. Simulation results show that the received signal reference power (RSRP) enhancement and performed handover, ping-pong, drop call, and outage probabilities reductions in CC-CADS outperformed the 3GPP's CADSs, thus leading to reduced interruption time, improved spectral efficiency, and seamless handover.
\end{abstract}

\section{Introduction}

In the recent 3GPP LTE-Advanced releases 10 and 11 (Rels. 10 and 11), five carrier aggregation deployment scenarios (CADSs) were proposed [1-4] to provide sufficient coverage and enhance system performance to support user mobility within cells. Each CADS provides a different coverage area, which depends on the operating frequency bands and antenna orientation of the configured CCs. Therefore, each CADS provides different system performance results for users through its mobility within the cells. If carrier aggregation (CA) technology is considered, one of these scenarios should be carefully selected through a mobility study. Selecting the appropriate CADS must be built on an in-depth study to improve system performance during user mobility. Thus, this paper attempts to achieve this target.

Few studies focus on system performance evaluation based on various CADSs in the LTE-Advanced system. In [5], the effect of CA on mobility was discussed over different CADSs in terms of handover delay, RSRP measurements, and handover trigger time used to trigger intra-LTE mobility when the neighbour becomes offset better than serving. In [6], a cell's average throughput was evaluated based on two different CADSs through user mobility. In [7], the secondary cell measurement period was relaxed according to CADS to save user equipment (UE) power, thus reducing handover failure probability. In $[8,9]$, three handover methods were proposed in selected CADS to address conventional difficult handover scheme problems. However, previous research did not consider all handover performance metrics such as spectral efficiency, interruption time, performed handover, ping-pong, drop call, and outage probabilities. These studies also did not cover this subject because of the evolution of CA technology in the LTE-Advanced system. Therefore, further investigation on handover performance based on different CADSs is required.

This paper proposes a coordinated contiguous-CADS (CC-CADS) that utilizes two CCs that operate on two frequencies located in a contiguous band. Each CC antenna is directed to a cell boundary of the other CC. CC-CADS aims 


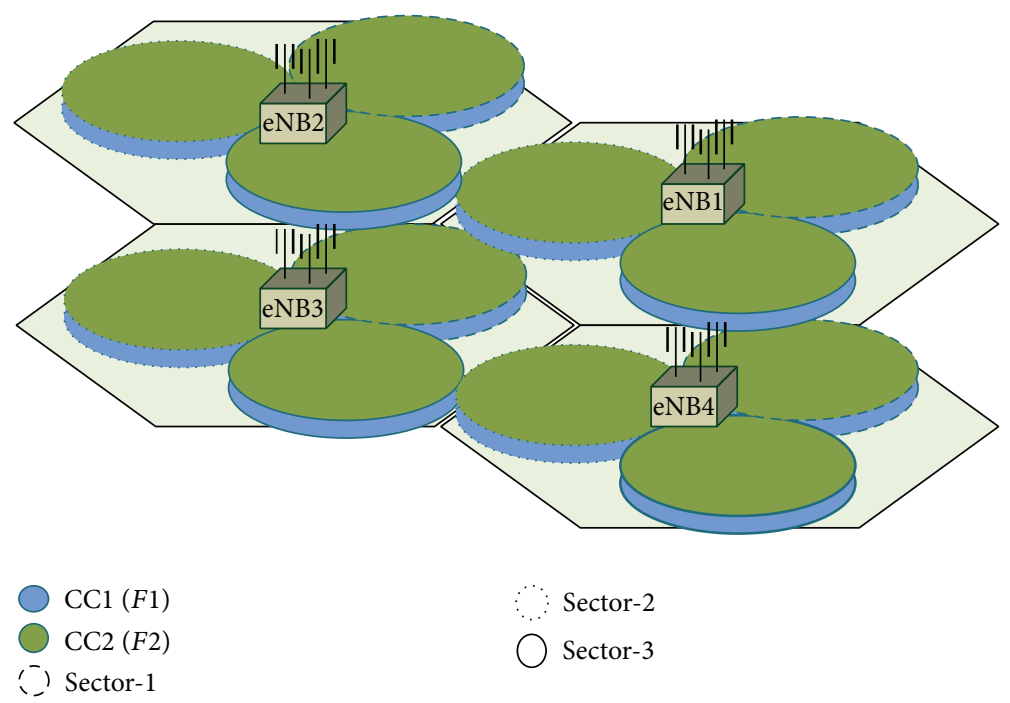

FIGURE 1: CADS1 with $120^{\circ}$ sectorization; each has two contiguous CCs in the same antenna direction.

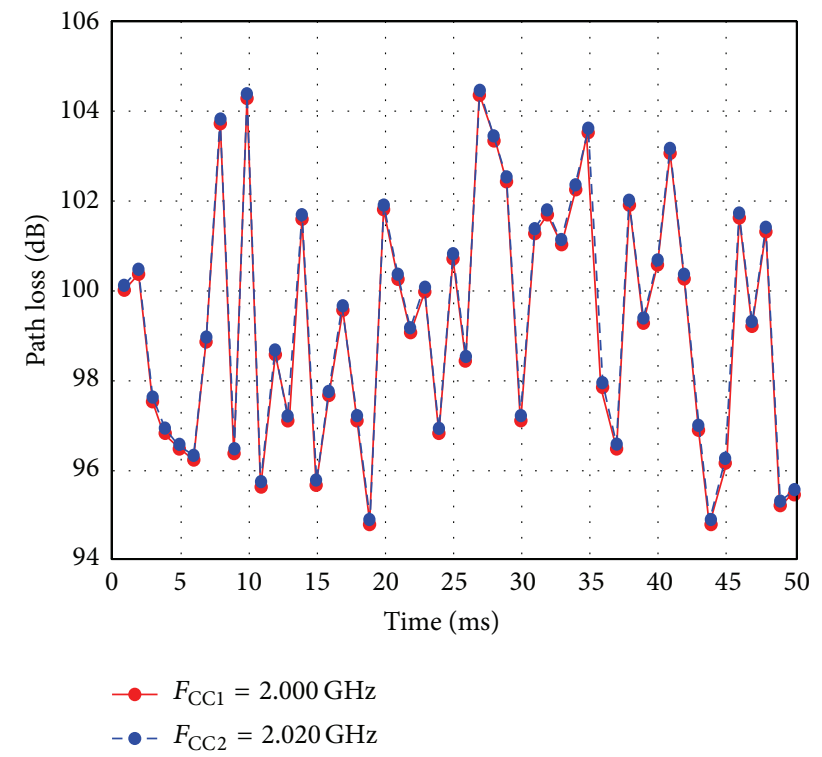

Figure 2: Path loss differences between CC1 and CC2 based on the CADS1.

to provide sufficient coverage to enhance spectral efficiency and decrease handover, ping-pong, drop calls, outage probabilities, and interruption time. The standard CADSs based on proposed 3GPP will be evaluated as background information for handover study in the CA environment. Identifying the suitable CADS that can be used for user mobility in the LTEAdvanced system is also beneficial.

The rest of this paper is organized as follows: Section 2 describes the standard CA deployment scenarios specified by 3GPP and the proposed CC-CADS is explained in Section 3. System and simulation models are presented in Section 4. The performance evaluation analysis is presented in Section 5. Results and discussions are presented in Section 6, followed by the conclusion in Section 7.

\section{CA Deployment Scenarios}

Five deployment scenarios (i.e., CADS1, CADS2, CADS3, CADS4, and CADS5) are proposed in the LTE-Advanced system as references to evaluate the CA performance through user mobility [1-4]. CADS1, CADS2, and CADS3 are considered in this paper to study the handover performance. CADS4 and CADS5 are not considered as they are included in the first three scenarios. CADS4 is a combination of CADS2 and CADS3, whereas CADS4 consists of nonuniform coverage based on different CCs, each with different antenna directions. Therefore, focusing on CADS1, CADS2, and CADS3 is sufficient to obtain performance results that are applicable for all five CADSs. For simplicity, each deployment scenario in this paper is based on two CCs only. One CC can be configured as a primary component carrier (PCC), whereas the other can be configured as the secondary component carrier (SCC). However, the total number of CCs that can be aggregated in the LTE-Advanced system is not limited to only two CCs and can reach up to a maximum of five CCs. The considered CADSs will be reviewed and explained in the following subsections.

2.1. First CADS (CADS1). In CADS1, the coverage areas of $\mathrm{CC} 1$ and CC2 cells are overlaid and colocated and are almost similar, as shown in Figure 1. These identical coverage areas of $\mathrm{CC} 1$ and $\mathrm{CC} 2$ are due to the similar path loss, where the colocated frequencies are assumed for both CCs and operate in a contiguous band. Therefore, the frequency difference between $F 1$ and F2 is almost negligible, which results in a low path loss difference between F1 and F2, as shown in Figure 2. Both CCs provide sufficient coverage and can support mobility. When both CCs are operating on 


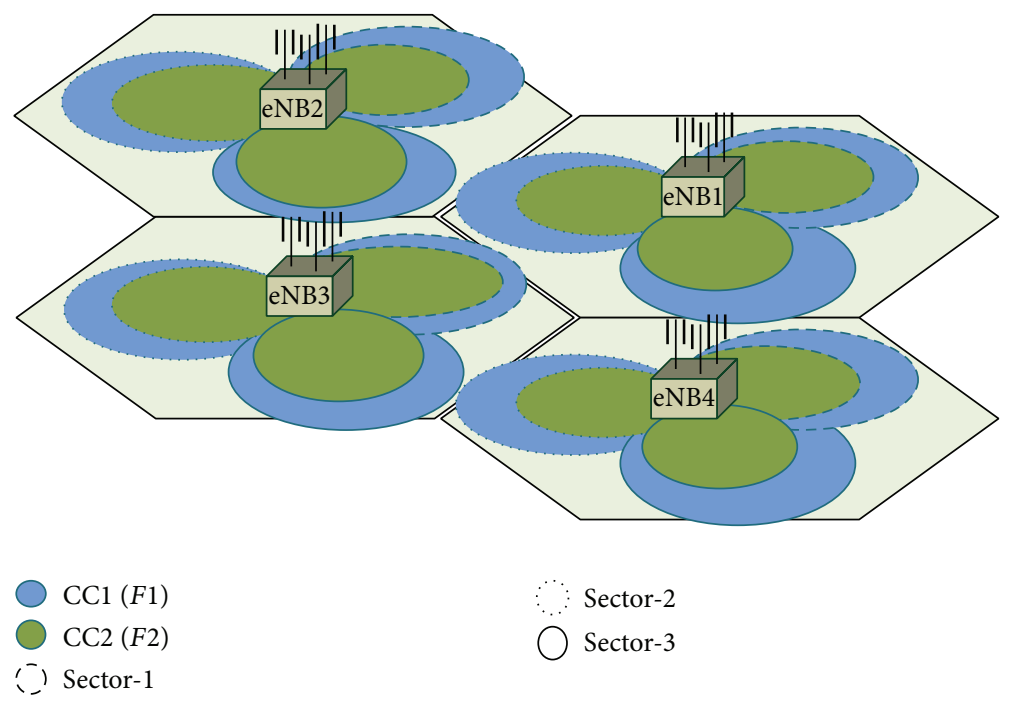

FIGURE 3: CADS2 with $120^{\circ}$ sectorization; each has two noncontiguous CCs in the same antenna orientation.

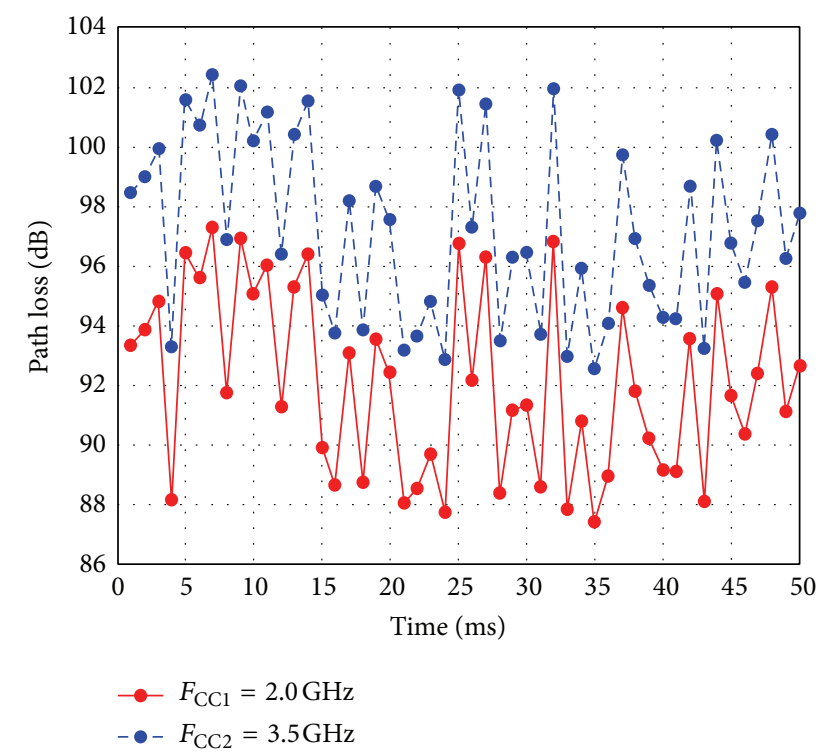

Figure 4: Path loss differences between CC1 and CC2 based on the CADS2.

a contiguous band and their antennas are directed in the same direction, aggregation is possible between the overlaid coverages of both CCs. However, in order to relate the coverage area with the path loss, the propagation model for macrocell urban area that is introduced in TR 36.942 [10] is considered in this paper. Whereas that model is appropriate for scenarios in the urban and suburban areas, where the height of buildings almost uniform, the propagation model is expressed by

$$
\begin{aligned}
L= & 40 \cdot\left(1-4 \cdot 10^{-3} \cdot \Delta h_{\mathrm{eNB}}\right) \cdot \log _{10}(d) \\
& -18 \cdot \log _{10}\left(\Delta h_{\mathrm{eNB}}\right)+21 \cdot \log _{10}\left(f_{c}\right)+80 \mathrm{~dB},
\end{aligned}
$$

where $d$ is the distance between eNB and served UE in kilometres and $f_{c}$ is the operating carrier frequency in
$\mathrm{MHz}$, while $\Delta h_{\mathrm{eNB}}$ represents the antenna height of eNB in metres. By considering the eNB antenna height of 15 metres above the average rooftop level and log-normally distributed shadowing of $\log _{10}(\varphi)$, the path loss (PL) model can be given by the following formula:

$$
\mathrm{PL}=58.8+37.6 \cdot \log _{10}(d)+21 \cdot \log _{10}\left(f_{c}\right)+\log _{10}(\varphi),
$$

where the random variable $\psi$ is expressed by $\psi=10^{\xi / 10}$, where $\xi$ is a zero mean, and standard deviation $(\sigma)$ is assumed to be $10 \mathrm{~dB}$. Moreover, considering Rayleigh fast fading $\vartheta_{\mathrm{dB}}$, the path loss model can be given by

$$
\begin{aligned}
\mathrm{PL}= & 58.8+37.6 \cdot \log _{10}(d)+21 \cdot \log _{10}\left(f_{c}\right) \\
& +\log _{10}(\varphi)+\vartheta_{\mathrm{dB}} .
\end{aligned}
$$

When $F 1$ and $F 2$ are operating in a contiguous band, the path loss difference will be very small, which can be neglected. In this paper, the operating frequencies of $F 1$ and $F 2$ start from $2 \mathrm{GHz}$. The bandwidth for each frequency is $20 \mathrm{MHz}$, whereas the spacing between two center frequencies is a multiple of $300 \mathrm{kHz}$ [1-9]. Consequently, the operating frequencies of F1 and $F 2$ are 2 and $2.0203 \mathrm{GHz}$, respectively.

2.2. Second CADS (CADS2). In CADS2, the coverage areas of CC1 and CC2 cells are overlaid and colocated, as shown in Figure 3. The path loss difference between CC1 and CC2 is mainly due to the large frequency difference between the operating frequencies of $\mathrm{CC} 1$ and $\mathrm{CC} 2$, as shown in Figure 4. The frequencies of $\mathrm{CC} 1$ and $\mathrm{CC} 2$ operate on different bands. $\mathrm{CC} 1$ operates on a lower frequency, whereas CC2 operates at a higher frequency. Therefore, $\mathrm{CC1}$ provides a stronger signal strength compared with CC2. Thus, CC1 has a good coverage area, whereas CC2 is employed to extend bandwidth to provide higher throughput to the users at close proximity to the eNB. Consequently, mobility management is performed based on the CCl coverage area only. When 


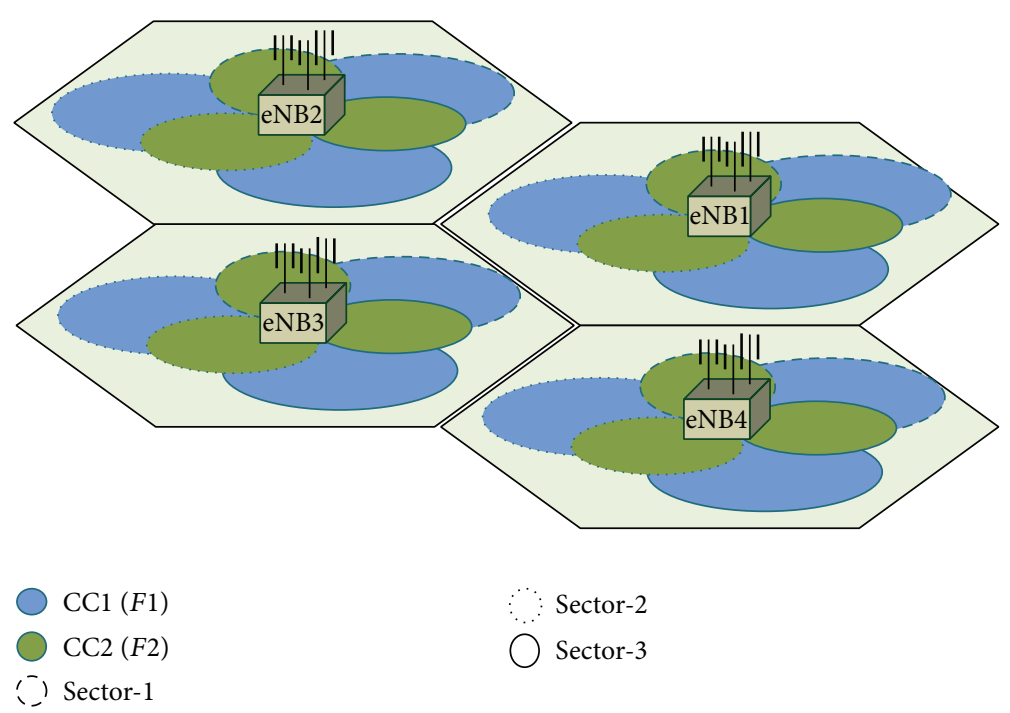

FIGURE 5: CADS3 with $120^{\circ}$ sectorization, each has two noncontiguous CCs with different antenna orientation.

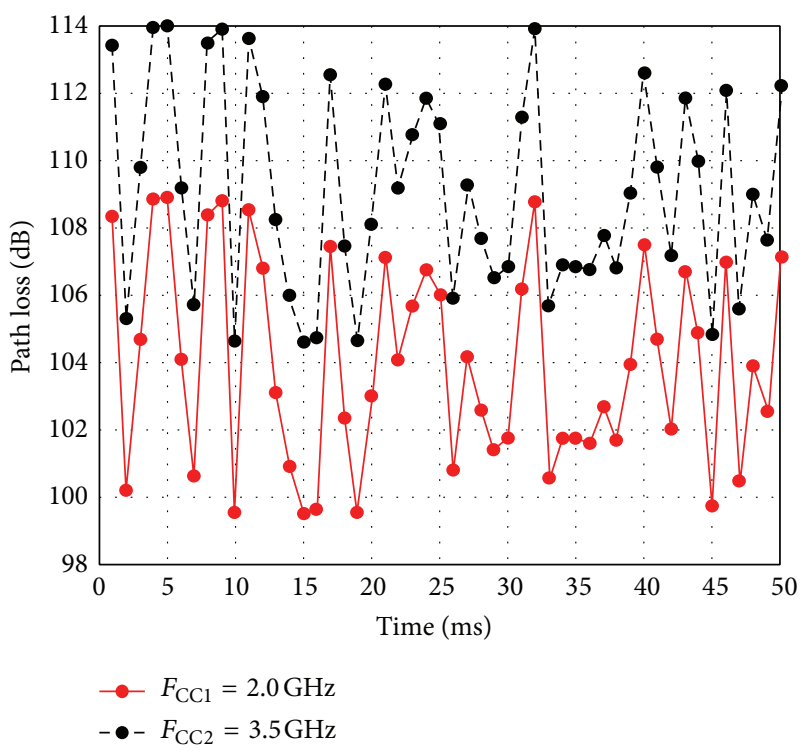

Figure 6: Path loss differences between CC1 and CC2 based on the CADS3.

two CCs operate on noncontiguous bands, aggregation can be observed between these two overlaid CCs.

2.3. Third CADS (CADS3). In CADS3, the coverage areas of $\mathrm{CC} 1$ and $\mathrm{CC} 2$ are colocated, as shown in Figure 5. In this scenario, the path loss difference between $\mathrm{CC} 1$ and CC2 is also large (as shown in Figure 6) because of the large difference between the operating frequency of $\mathrm{CC} 1$ and $\mathrm{CC} 2$, which operate on noncontiguous bands. However, CC1 always operates on a lower frequency to provide a larger coverage area than CC2, whereas CC2 operates at a higher frequency that results in a smaller coverage area than $\mathrm{CC1}$ because of the larger path loss from CC2. Thus, mobility support can be effectively performed based on the $\mathrm{CCl}$ coverage area, whereas CC2 is used to provide high throughput to the users. The CC2 antenna is also directed to the cell boundary of CC1 to enhance cell edge throughput [6].

\section{Proposed CC-CADS}

This paper proposes a new CC-CADS based on two CCs that operate on two frequencies in a contiguous band, as shown in Figure 7(a). The antenna direction of each CC is directed to the cell boundary of the other CC, as illustrated in Figure 7(b). The path loss differences between these two CCs are neglected, as shown in Figure 8. This identical path loss results from both CCs (CC1 and CC2) operating in contiguous bands, which provide equivalent coverage areas around the serving eNB. Thus, the proposed CC-CADS can provide sufficient coverage area around serving eNB, which can enhance system performance to support user mobility within the cells. In practice, the most likely scenario would be to deploy CC1 and CC2 in the same band with their directional antenna pointed in different directions. Both components can be aggregated at the same eNB.

\section{System Models and Simulation Scenarios}

4.1. System Model. The LTE-Advanced system is modeled on 3GPP specifications [10] by considering the 61 macrohexagonal cell layout model, as shown in Figure 9. Each hexagonal cell is built with an intersite distance of $500 \mathrm{~m}$, with one eNB located at its center. Each hexagonal cell consists of three sectors with two directional antennas in each sector. With regard to the users, at the initiation cycle of the simulation, 50 UEs are generated randomly in the centre eNB. Then from the second cycle, random numbers of UEs are generated and removed randomly at random positions in each cell. This random generation and removal of UEs are intended to mimic a random generation of traffic throughout 


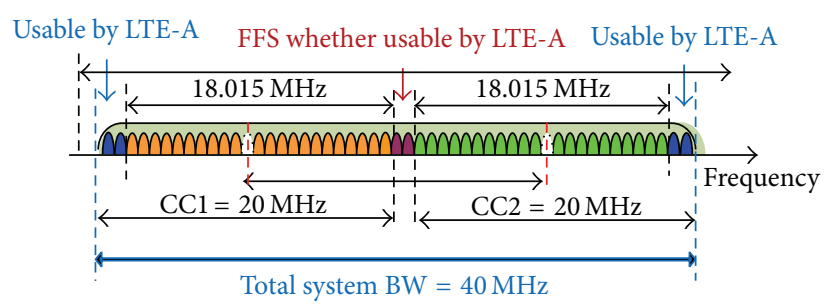

(a) Assumed operating frequency bands in the proposed CC-CADS based on two contiguous bands
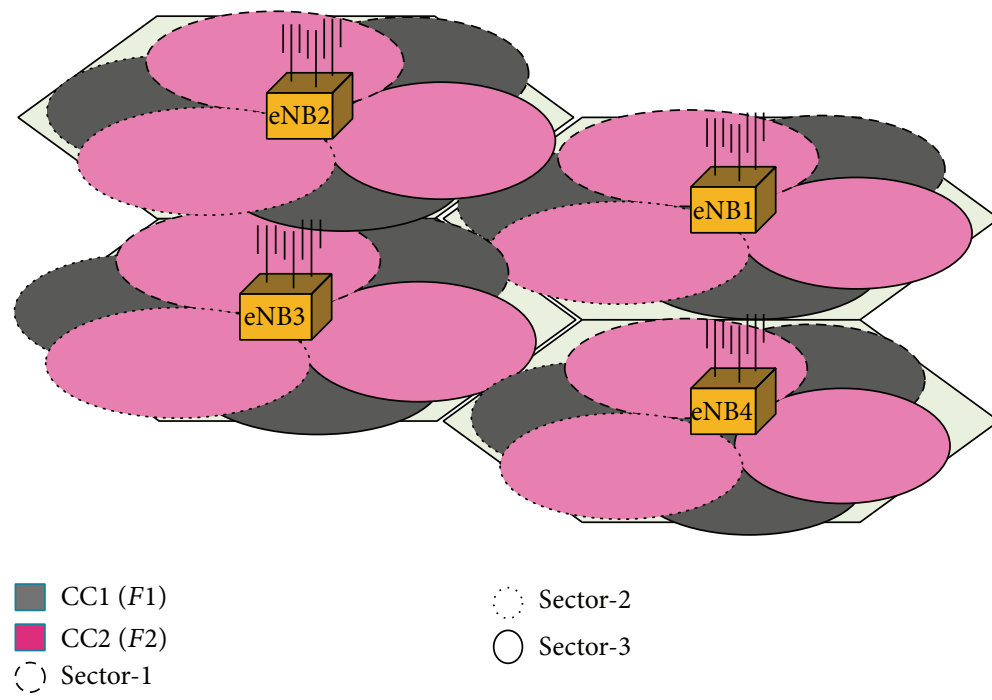

(b) Proposed CC-CADS with $120^{\circ}$ sectorization; each based on two contiguous CCs with different antenna directions

FIgURE 7: Coordinated contiguous CA deployment scenario.

the simulation. The mobility model is considered to be a random mobility model (RMM) for all UEs; thus all UEs' directional movements are selected randomly with a fixed speed throughout the simulation with five different mobile speeds (i.e., 30, 60, 90, 120, and $140 \mathrm{~km} / \mathrm{hr}$ ).

This paper considers four CA deployment scenarios. In CADS1 and in the proposed CC-CADS, the operating frequencies for $\mathrm{CC} 1$ and $\mathrm{CC} 2$ are 2 and $2.0203 \mathrm{GHz}$, respectively. In CADS2 and CADS 3, the operating frequencies for $\mathrm{CC1}$ and CC2 are 2 and $3.5 \mathrm{GHz}$, respectively. All these operating frequencies are assumed based on the agreed band scenarios for Rels. 10 and 11 timeframe [10, 11]. As for frequency planning, a unity frequency reuse factor is assumed for both CCs' overall CA deployment scenarios.

In CADS1 and CADS2, both CCs in each sector are directed to the same flat side of the hexagon cell with the same beam angle, as illustrated in Figures 10(a) and 10(b), respectively. The beam angles for $\mathrm{CC} 1$ and $\mathrm{CC} 2$ antennas in sectors 1,2 , and 3 are $45^{\circ}, 180^{\circ}$, and $300^{\circ}$ degrees, respectively. In CADS3 and CC-CADS, each CC antenna is directed toward a different flat side of the hexagon cell, as illustrated in Figures 10(c) and 10(d), respectively. Consequently, the beam direction of each antenna results in a different beam angle. Specifically, the $\mathrm{CCl}$ antenna in sectors 1,2 , and 3 is directed with beam angles of $30^{\circ}, 150^{\circ}$, and $270^{\circ}$ degrees, respectively, whereas the beam directions of CC2 antenna in sectors 1,2 , and 3 are directed with beam angles of $90^{\circ}, 210^{\circ}$, and $330^{\circ}$ degrees, respectively.

The transmitted power from each eNB over each CC is the same. The interference signals are considered from six neighbouring eNBs located in the first tier around the serving eNB during the simulation time. Since the served UEs in the serving eNB are located further away from the neighbouring eNBs, those are located in the second tier; thus the received signals by served UEs will be very low from those eNBs. Therefore, the interference signals from the second tier neighbouring eNBs around the serving eNB are very weak, which can be neglected. The adaptive modulation and coding (AMC) scheme is employed to modulate the signal based on the sets of modulation schemes and coding rate introduced in [12-14]. Radio link failure (RLF) detection,radio resource control (RRC) reestablishment procedure, and nonaccess stratum (NAS)-recovery procedures are considered in the simulation. The essential parameters used in this study listed in Table 1 are based on the LTE-Advanced (Rel. 11) system profiles that have been defined by 3GPP specifications in $[10-15]$.

4.2. Simulation Scenario. The user establishes a setup connection with the serving eNB by using the best selected CC configured as a PCC. The PCC can be changed by 
TABLE 1: Simulation parameters.

\begin{tabular}{|c|c|}
\hline Parameter & Assumption Value \\
\hline Cellular Layout & Hexagonal grid, 61 cell sites, 3 Sectors per cell site, 2 CCs per Sector \\
\hline Minimum Distance between UE and eNB & 35 meters \\
\hline Total eNB TX Power & $46 \mathrm{dBm}$ per $\mathrm{CC}$ \\
\hline Shadowing Standard Deviation & $8 \mathrm{~dB}$ \\
\hline White Noise Power Density (Nt) & $-174 \mathrm{dBm} / \mathrm{Hz}$ \\
\hline eNBs Noise Figure & $5 \mathrm{~dB}$ \\
\hline Thermal Noise Power (Np) & $\mathrm{Np}=\mathrm{Nt}+10 \log (\mathrm{BW} \times 106) \mathrm{dB}$ \\
\hline UE Noise Figure & $9 \mathrm{~dB}$ \\
\hline Operation Carrier Bandwidth & $20 \mathrm{MHz}$ (PCC and SCC) \\
\hline Total System Bandwidth & $40 \mathrm{MHz}(2 \mathrm{CC} s \times 20 \mathrm{MHz})$ \\
\hline Carriers Frequencies & $2 \mathrm{GHz}, 2.023 \mathrm{GHz}$ and $3.5 \mathrm{GHz}$ \\
\hline Number of Physical Resource Block (PRBs) per CC & $100 \mathrm{PRBs} / \mathrm{CC}$ \\
\hline Number Subcarriers/PRB & 12 Subcarriers per FRB. \\
\hline Number of OFDM Symbols per Subframe & 7 \\
\hline Sub-carrier Spacing & $15 \mathrm{kHz}$ \\
\hline Resource Block Bandwidth & $180 \mathrm{kHz}$ \\
\hline Q_rxlevmin & $-101.5 \mathrm{dBm}$ \\
\hline Measurement Interval & $50 \mathrm{~ms}$ for PCC and SCC \\
\hline Time-to-Trigger & $320 \mathrm{~ms}$ \\
\hline Handover Margin (HOM) & $6 \mathrm{~dB}$ \\
\hline Each X2-interface delay & $10 \mathrm{~ms}$ \\
\hline Each eNB Process Delay & $10 \mathrm{~ms}$ \\
\hline T311 & $10 \mathrm{~s}$ \\
\hline Simulation Time & $330 \mathrm{~s}$ \\
\hline
\end{tabular}

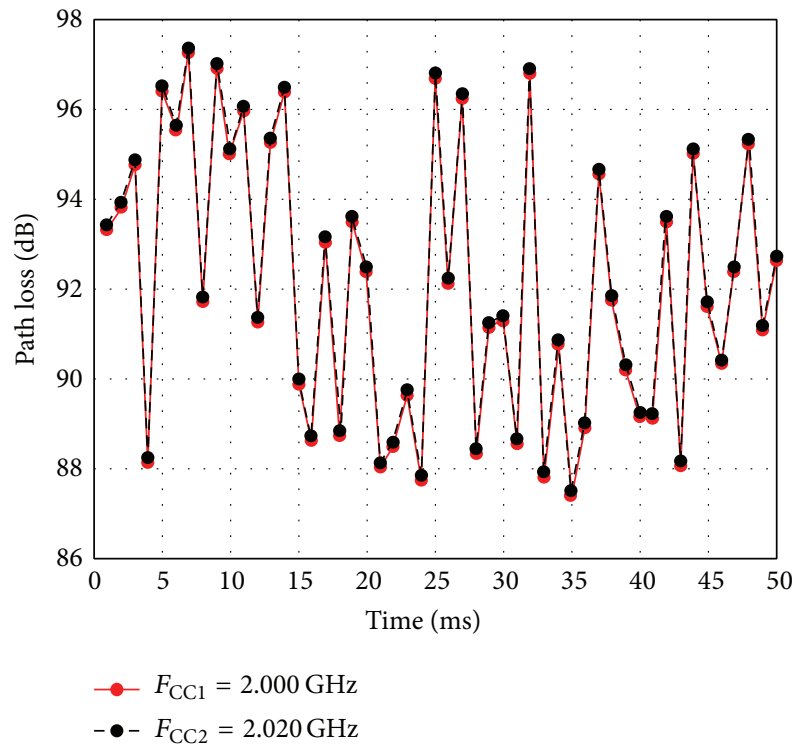

FIgURE 8: Path loss differences between $\mathrm{CC} 1$ and $\mathrm{CC} 2$ based on the proposed CC-CADS.

performing the handover procedure to the SCC. The user moves randomly within the cell and measures the RSRP from all the neighbouring eNBs periodically at every trigger time. The user then sends a measurement report to the serving

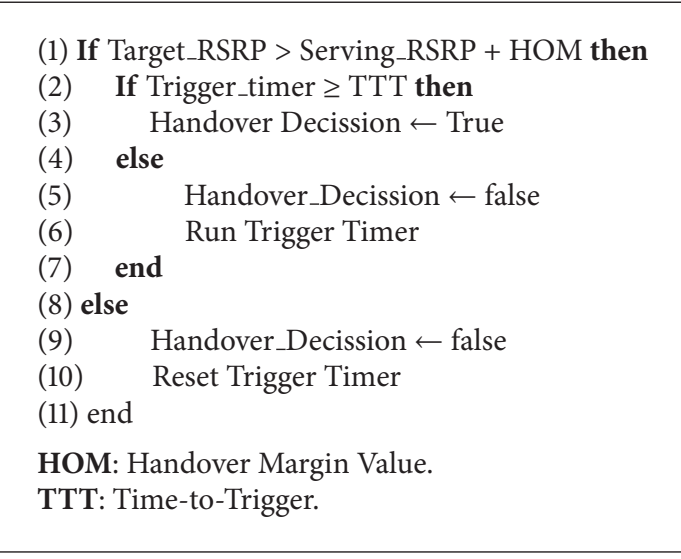

Algorithm 1: Handover decision algorithm.

eNB. The serving eNB selects the best eNB as the target eNB based on the measured RSRP level. Subsequently, it makes a handover decision based on the best selected target eNB. The handover decision is taken based on the serving RSRP level over the PCC, handover margin, time-to-trigger (TTT), and the selected target RSRP level. The handover decision can be illustrated by Algorithm 1 .

Once the handover decision is validated, the serving eNB starts to initiate a handover procedure with the target eNB. The handover sequence is performed based on the 
TABLE 2: Handover model descriptions.

\begin{tabular}{lc}
\hline HO-Model-No. & Handover Models Description \\
\hline HO-Model-1 & CC1 $\rightarrow$ CC2, Sector 1 $\rightarrow$ Sector 1, Same eNB1 \\
HO-Model-2 & CC2 $\rightarrow$ CC2, Sector 1 $\rightarrow$ Sector 2, Same eNB1 \\
HO-Model-3 & CC2 $\rightarrow$ CC1, Sector 2 $\rightarrow$ Sector 3, Same eNB1 \\
HO-Model-4 & CC1 $\rightarrow$ CC1, Sector 3 $\rightarrow$ Sector 2, eNB1 $\rightarrow$ eNB2 \\
HO-Model-5 & CC2 $\rightarrow$ CC1, Sector 2 $\rightarrow$ Sector 1, eNB2 $\rightarrow$ eNB3 \\
\hline
\end{tabular}

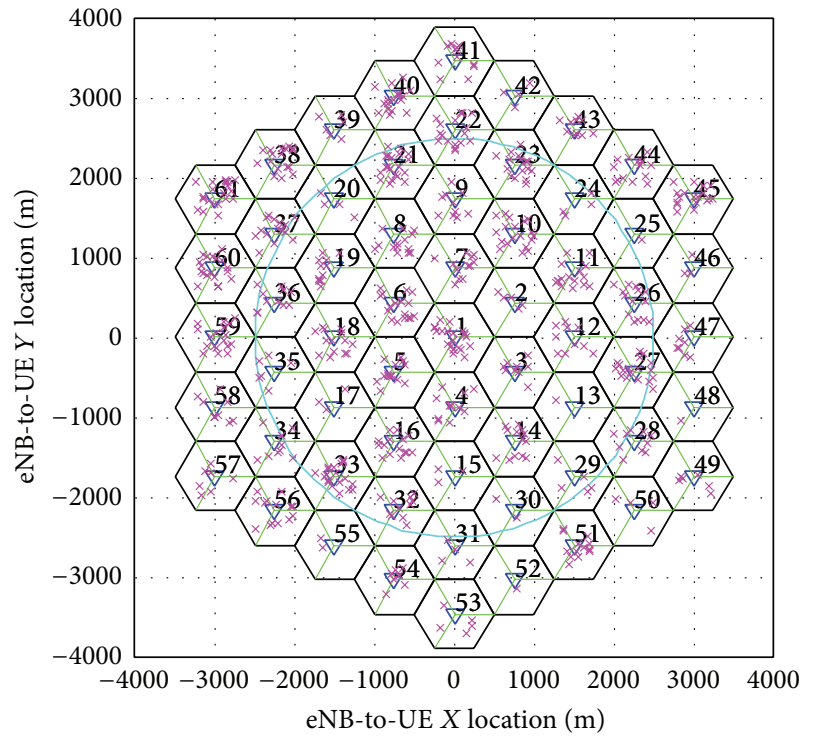

$\begin{array}{ll}\times & \mathrm{UE} \\ \nabla \mathrm{eNB}\end{array}$

FIGURE 9: LTE-Advanced system model.

LTE-Advanced system handover procedure described in [15]. The handover process is initiated when the target eNB establishes admission control (AC) after the handover request is received. When the target $\mathrm{eNB}$ has verified the $\mathrm{AC}$ request, it acknowledges the handover request to the serving eNB. The downlink (DL) resource allocation process is then activated. The handover to the target eNB is executed when the required parameters are received by the UE contained in the RRCconnection-reconfiguration message.

The RSRP radio link connection status is monitored and updated periodically regardless of a handover request being initiated. An RRC reestablishment request is initiated if a RLF occurs and is sent to the target eNB. Cell reselection and recovery occur in line with the T311 period, which is the maximum allowed time for connection recovery through the RRC reestablishment procedure. The UE searches the RSRP and selects a target cell if it fulfills the minimum required signal level (Qrxlevmin). If multiple cells are available, the best cell is selected by the UE, and it then sends a reestablishment request that initiates the connection reconfiguration. If no cell is identified by the UE within the T311 period and no RRC reestablishment occurs, the NAS recovery procedure takes over.
In case $\mathrm{RRC}$ reestablishment fails, the UE continues to identify a suitable cell in the target eNB throughout the NAS recovery procedure. Even after the T311 maximum time has expired, the search is repeated until a suitable cell is identified and reconnection takes place. In our simulation model, recovery events were considered and failed events were recorded in the User-plane (U-plane) interruption time to improve performance evaluation. This approach allows a better comparison of performance in terms of serving RSRP, throughput, interruption time, handover, ping-pong, call drop, and outage probabilities when comparing this model to CA handover technique in an actual network.

4.3. Handover Scenarios. The advent of CA technology in the LTE-Advanced system Rel. 10 or Rel. 11 has increased the handover rates to more than the handover occurrence in LTE (Rels. 8 and 9). In the LTE system (Rels. 8 and 9), handover occurs between different eNB cells or across sectors of the same cell. In the LTE-Advanced system, the handover procedure can occur within the same sector between two frequencies $[15,16]$. Therefore, a total of five handover models are possible in the LTE-Advanced system: (i) interfrequency, intrasector, and intra-eNB handover (HO-Model1); (ii) intrafrequency, intersector, and intra-eNB handover (HO-Model-2); (iii) interfrequency, intersector, and intraeNB handover (HO-Model-3); (iv) intrafrequency, intereNB handover (HO-Model-4); and (v) interfrequency, intereNB handover (HO-Model-5). The details of these handover models are shown in Figure 11 and described in Table 2. Intrafrequency means the serving and target carriers are the same, whereas interfrequency means the serving and target carriers are different. Handovers between sectors can be intrasector or intersector depending on whether the target and serving sectors are the same or different, respectively. If the target and serving eNBs are the same, the handover is called intra-eNB, whereas the handover is called inter-eNB when the target and serving eNBs are different. All these handover models are considered in this study.

\section{Performance Evaluation}

5.1. Downlink RSRP and SINR Evaluation. The DL transmission signals based on the CA technique and OFDMA scheme in the LTE-Advanced environment is considered in this paper. The serving eNB is assumed to be able to serve each user by $N_{\mathrm{sc}}^{\mathrm{UE}}$ subcarriers over $N_{\mathrm{CC}}^{\mathrm{UE}}$ CCs assigned to each user simultaneously. This paper considers the definition of PRB introduced by 3GPP in TS.36.201 [14]. If the total 


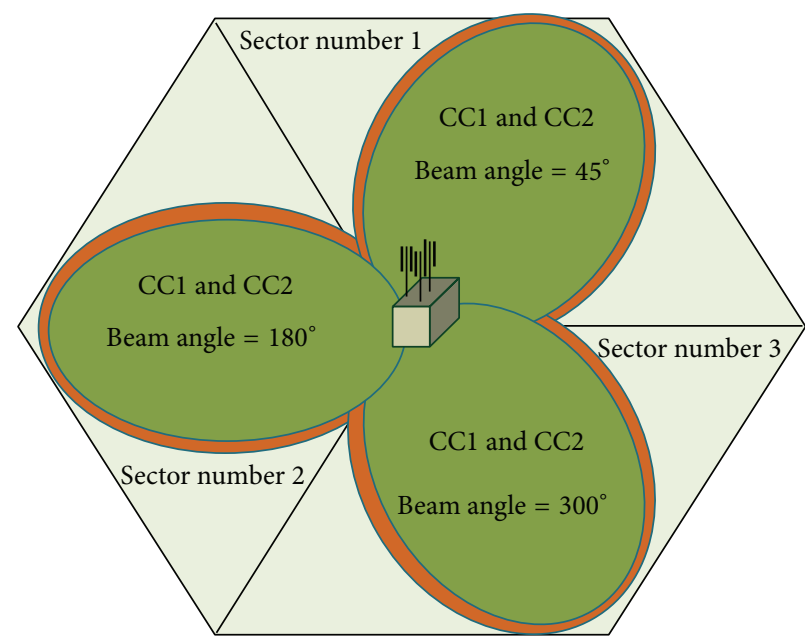

(a)

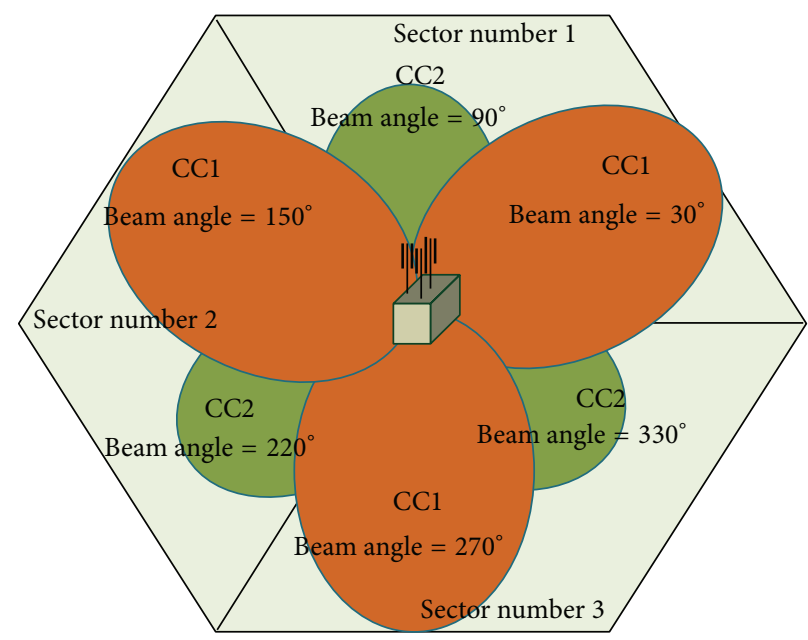

(c)

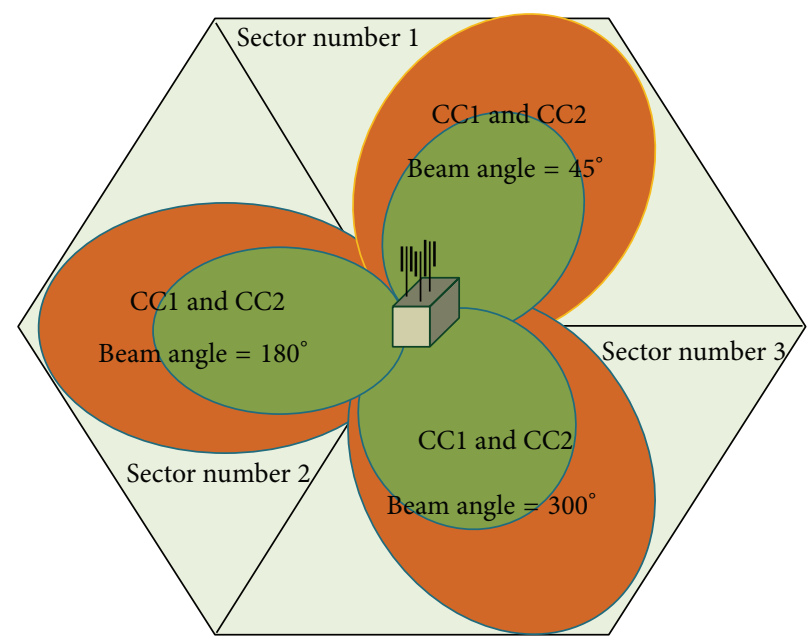

(b)

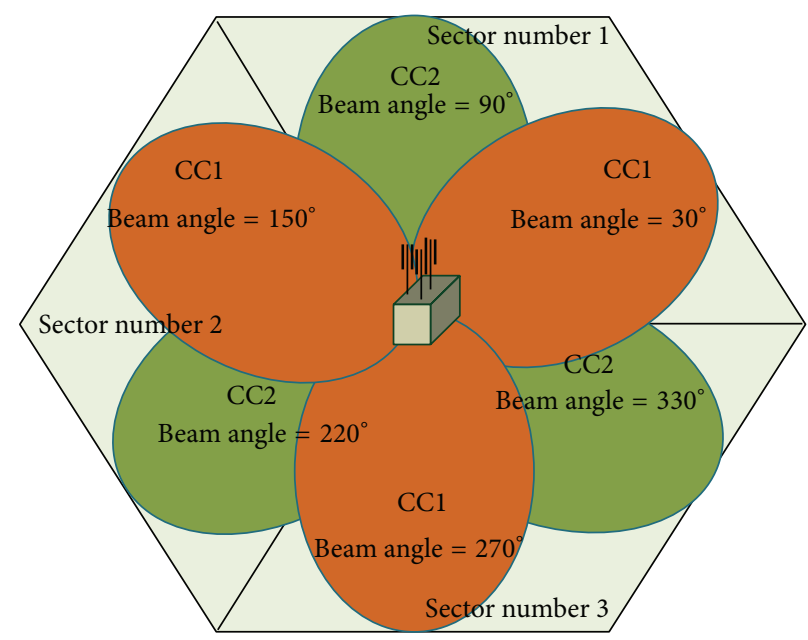

(d)

Figure 10: CC1 and CC2 beam directions for (a) CADS1, (b) CADS2, (c) CADS3, and (d) CC-CADS.

subcarriers over a single CC are expressed by $N_{\mathrm{sc}}^{\mathrm{CC}}$, the total transmission power $P_{t}$ is equally distributed among all the available subcarriers $N_{\mathrm{sc}}^{\mathrm{CC}}$. Thus, the total transmission power over each subcarrier can be expressed by the following [17]:

$$
P_{\mathrm{tsc}}=\frac{P_{t}}{N_{\mathrm{sc}}^{\mathrm{CC}}}
$$

The transmitted power over any subcarrier $P_{\text {tsc }}$ from any eNB is the same over any CC. Therefore, the desired received signal power $P_{\text {rsc }_{i, n, k, x}}$ by UE $j$ on subcarrier $k$ over CC $n$ at position $x$ in the DL transmission can be expressed as follows:

$$
P_{\mathrm{rsc}}, j, k, k, x=P_{\mathrm{tsc}} \cdot g_{t} \cdot g_{r} \cdot \ell \cdot \varphi \cdot \vartheta
$$

where $P_{\text {rsc }_{j, n, k, x}}$ and $P_{\mathrm{tsc}}$ represent the desired received and transmitted signal power over one subcarrier, respectively. The notations $g_{t}$ and $g_{r}$ represent the transmitter and receiver antenna gains, respectively. $\ell, \varphi$, and $\vartheta$ represent loss, lognormally distributed shadowing, and Rayleigh fast fading, respectively. Consequently, the signal-to-interference-plusnoise-ratio (SINR) at UE $j$ on subcarrier $k$ over CC $n$ at position $x$ can be expressed by the following:

$$
\operatorname{SINR}_{j, n, k, x}=\frac{P_{\mathrm{tsc}} \cdot g_{t} \cdot g_{r} \cdot \ell \cdot \varphi \cdot \vartheta}{\sum_{i=1}^{H} P_{\text {int }_{-j, k, n, x, i}}+\operatorname{Pno}_{j, n, k, x}},
$$

where $\sum_{i=1}^{H} P_{\text {int }}{ }_{-i, k, n, x, i}$ is the total interferences to UE $j$ on a subcarrier $k$ over component carrier $n$ at position $x$. These interferences are received by UE $j$ from $H$ neighbors eNBs, which are located at the first tier around the serving eNB. Pno $_{j, n, k, x}$ is the noise power for user $j$.

5.2. Handover Probability. Handover probability (HOP) is a significant performance indicator, which is used to measure quality of service. This metric represents the average number 


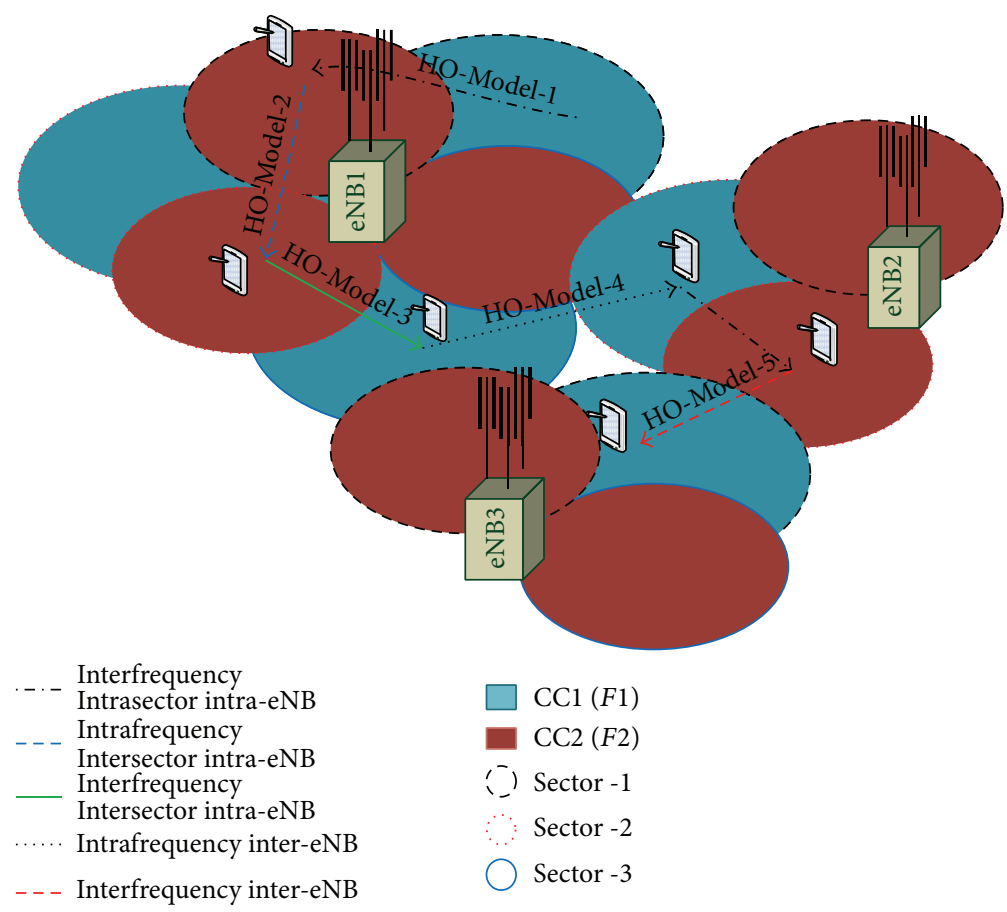

FIGURE 11: Different handover model scenarios.

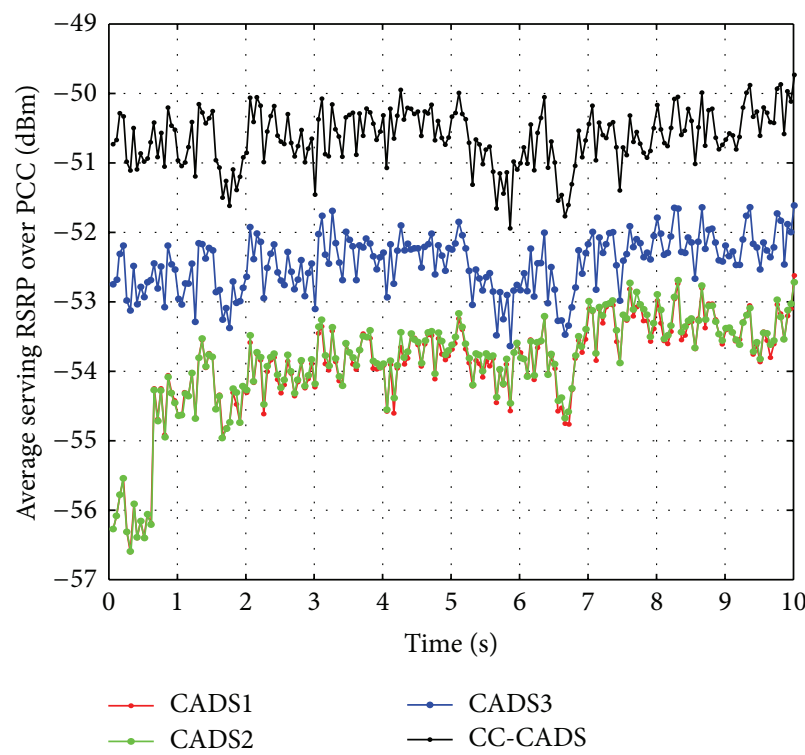

(a) Serving RRSP versus time

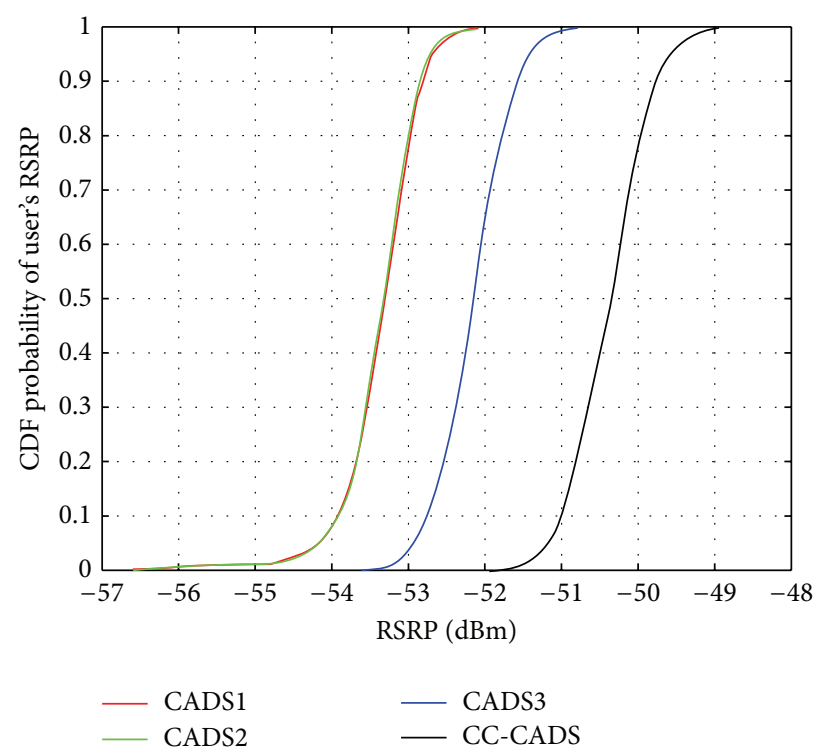

(b) The CDF of serving RRSP

FIGURE 12: Serving RSRP performance based on different CADSs.

of handovers that can be performed per call. It can be expressed by

$$
\mathrm{HOP}=\frac{N_{\mathrm{HO}}^{\mathrm{UE}_{i}}}{N_{\text {Call }}^{\mathrm{UE}}}
$$

where $N_{\mathrm{HO}}^{\mathrm{UE}_{i}}$ represents the total number of handover for $\mathrm{UE}$ $i$ over all calls, while $N_{\text {Call }}^{\mathrm{UE}_{i}}$ is the total number of calls of $\mathrm{UE} i$ throughout the simulation time.
5.3. Handover Ping-Pong Probability. Handover ping-pong probability (HOPPP) is important metrics through the study of handover, which is used to measure the number of unnecessary handover that can be frequently performed between two adjacent cells. In other words, the handover can be considered as ping-pong if UE_ $i$ leave the serving eNB_A to the target eNB_B and then handed back to the serving eNB-A in a period less than a specific interval of time, which is called a critical time $T_{\text {critical }}$. So, if handover takes a place, 


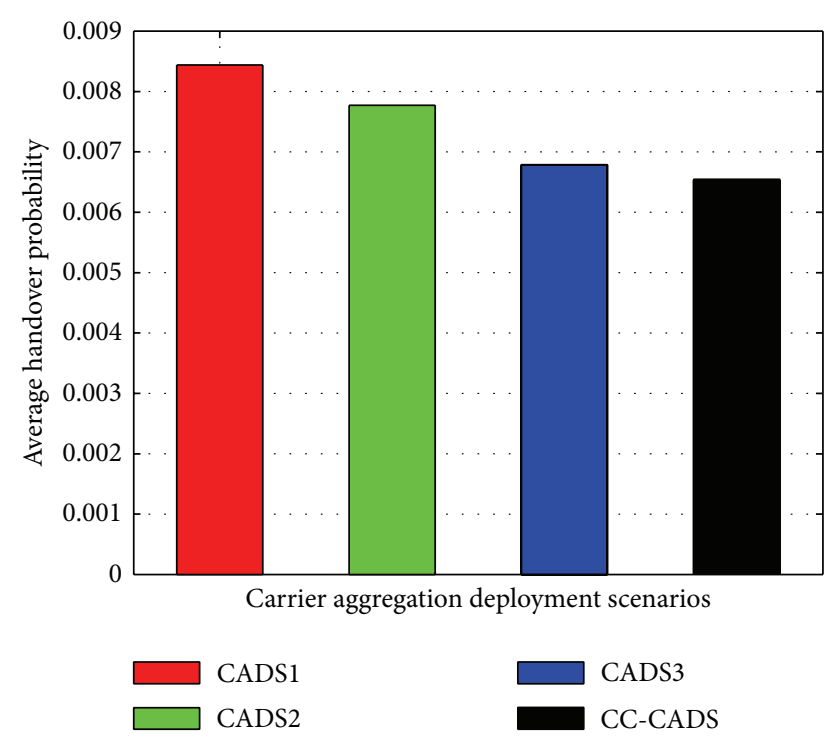

FIGURE 13: Handover probability based on different CADSs.

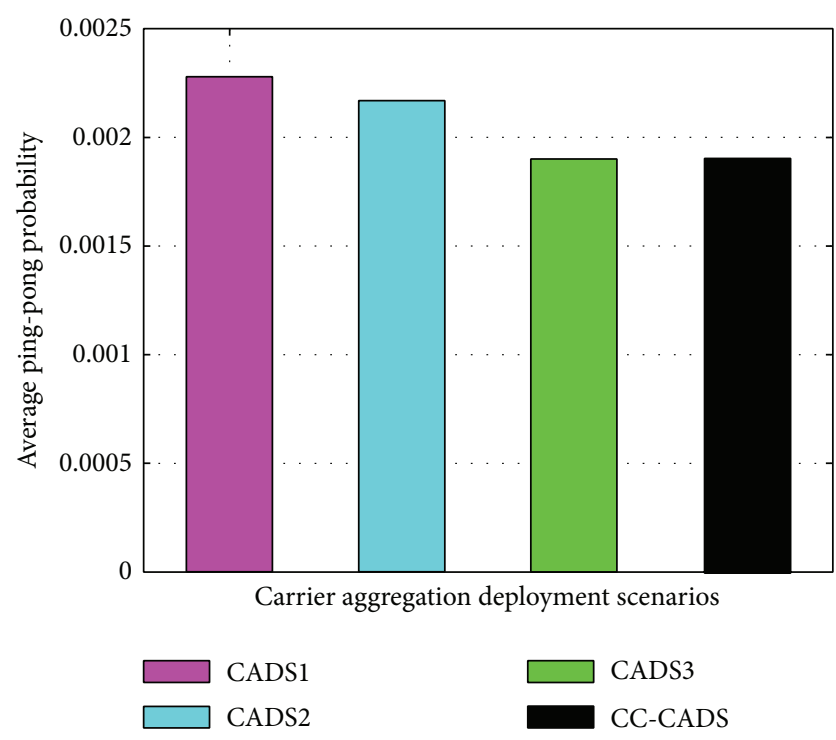

FIGURE 14: Handover ping-pong probability based on different CADSs.

the HOPPP can be estimated by the following probability expression:

$$
P_{\text {HOPPP }}=P\left[T_{\text {Interval }} \leq T_{\text {critical }}\right] \text {, }
$$

where $T_{\text {Interval }}$ represents the time interval between the leaving time from the serving eNB-A and the trigger handed back time to the same eNB-A. It can be easily expressed by

$$
T_{\text {Interval }}=T_{\text {Leave }}-T_{\text {handed back }} \text {, }
$$

where $T_{\text {Leave }}$ represents the leaving time from the serving eNB-A, while $T_{\text {handed back }}$ represents the handed back time to the serving eNB-A. Thus, if UE is handed back to the old serving eNB (eNB-A) and $T_{\text {Interval }}$ is less than $T_{\text {critical }}\left(T_{\text {Interval }}<\right.$
$\left.T_{\text {critical }}\right)$, handover ping-pong is recorded. Consequently, the average handover ping-pong probability can be represented by

$$
\mathrm{HOPPP}=\frac{N_{\mathrm{HOPP}}^{\mathrm{UE}_{i}}}{N_{\mathrm{HO}}^{\mathrm{UE}_{i}}},
$$

where $N_{\mathrm{HOPP}}^{\mathrm{UE}_{i}}$ represents the total number of handovers that are recorded as ping-pong for UE $i$ over all calls, while $N_{\mathrm{HO}}^{\mathrm{UE}_{i}}$ represents the total number of handover for UE $i$ throughout the simulation time.

5.4. Drop Call Probability. Drop call probability (DCP) is one of the key performance indicators that are used to measure quality of service. It basically measures the probability of dropping calls before the handover procedure is completed or if the radio link connection fails after the connection to the serving eNB is established. DCP can be evaluated by the following expression:

$$
\mathrm{DCP}=\frac{\mathrm{DCR}}{\mathrm{TCR}}
$$

where DCR represents the total number of dropped calls rate in the cell, while TCR is the total call rate, which represents the total number of established call in the cell.

5.5. Interruption Time. Interruption time is measured as the duration from which the U-plane is stopped until the U-plane is resumed. The U-plane is stopped if RLF is detected, the transmission of $\mathrm{HO}$ command is started, or radio link control retransmission is exceeded. The $\mathrm{U}$-plane can be resumed if the $\mathrm{HO}$ complete status, $\mathrm{RRC}$ reestablishment procedure, or NAS recovery procedure is successfully completed.

5.6. Downlink Spectral Efficiency. Spectral efficiency represents the average bits per second per Hertz (bps/Hz) that can be transmitted over a radio link channel and received correctly at the receiver side. The user's spectral efficiency can be represented mathematically by aggregating the total user's throughput that is correctly received by the user at a specific time and divided by the total user's channel bandwidth. Therefore, the normalized spectral efficiency $\eta_{j}$ for user $j$ can be expressed by the following formula [12]:

$$
\eta_{j}=\frac{\gamma}{T_{j} \times \omega_{\mathrm{BW}}^{\mathrm{UE}}}(\mathrm{bps} / \mathrm{Hz}),
$$

where $\gamma$ denotes the number of correctly received bits for user $j$ and $T_{j}$ represents the time when the data bits are received for the user $j$. $\omega_{\mathrm{BW}}^{\mathrm{UE}}$ denotes the user's channel bandwidth that can be calculated by the following:

$$
\omega_{\mathrm{BW}}^{\mathrm{UE}}=N_{\mathrm{RB}}^{\mathrm{UE}} \times B_{\mathrm{RB}},
$$

where $N_{\mathrm{RB}}^{\mathrm{UE}}$ denotes the number of PRBs assigned to UE $j$ and $B_{\mathrm{RB}}$ denotes the PRB's bandwidth. 


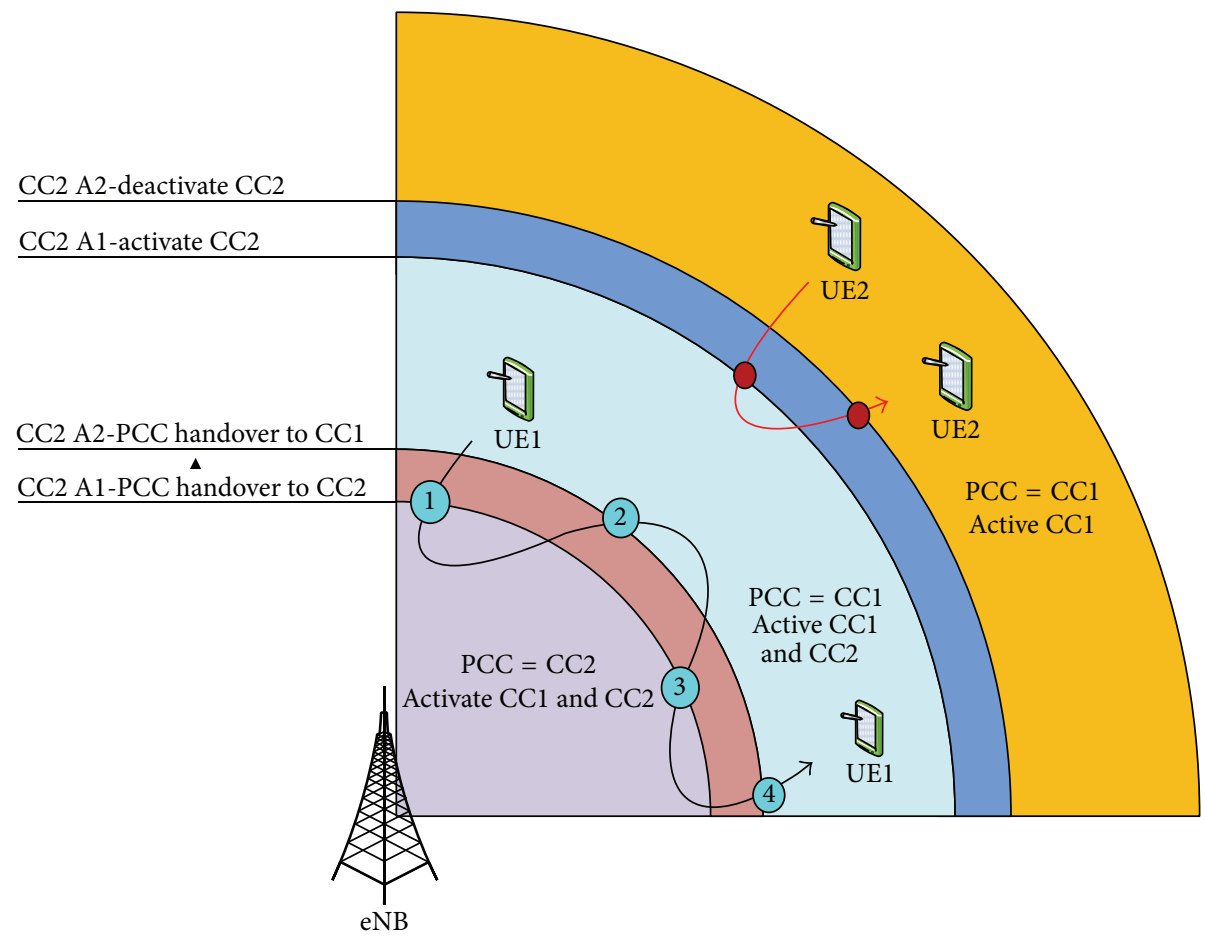

FIGURE 15: Handover probability between CC1 and CC2 based on CADS2.

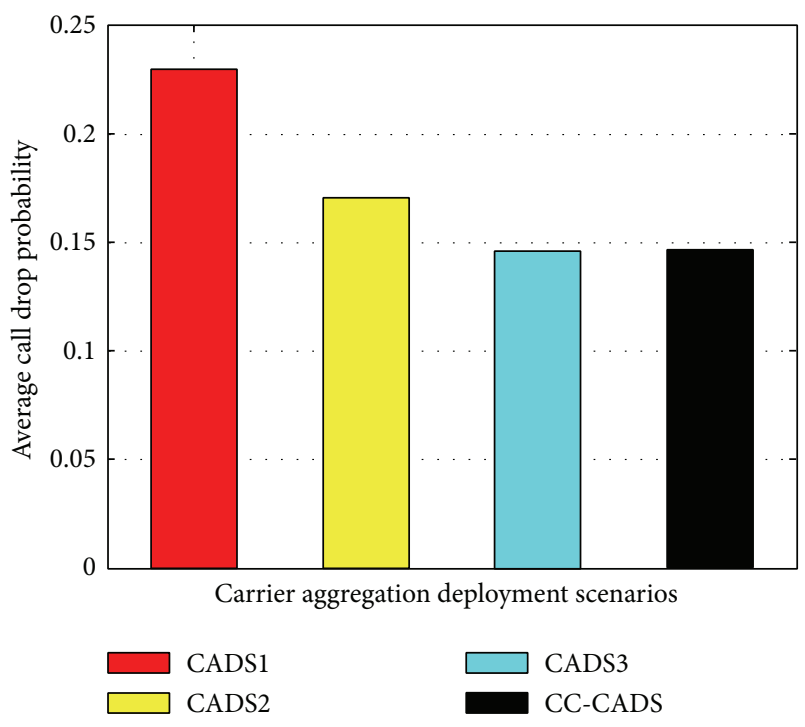

FIGURE 16: Drop call probability based on different CADSs.

The total correctly received user's throughput over one CC through a subframe time $\left(T_{j}\right)$ can be expressed by the following mathematical formula [18]:

$$
R_{\mathrm{bit}}^{\mathrm{UE}}=\frac{N_{\mathrm{RB}}^{\mathrm{UE}} \times N_{\mathrm{sc}}^{\mathrm{RB}} \times N_{\mathrm{symb}}^{\mathrm{sc}} \times m_{\mathrm{bit}}^{\mathrm{symb}}}{T_{j}} \mathrm{CR},
$$

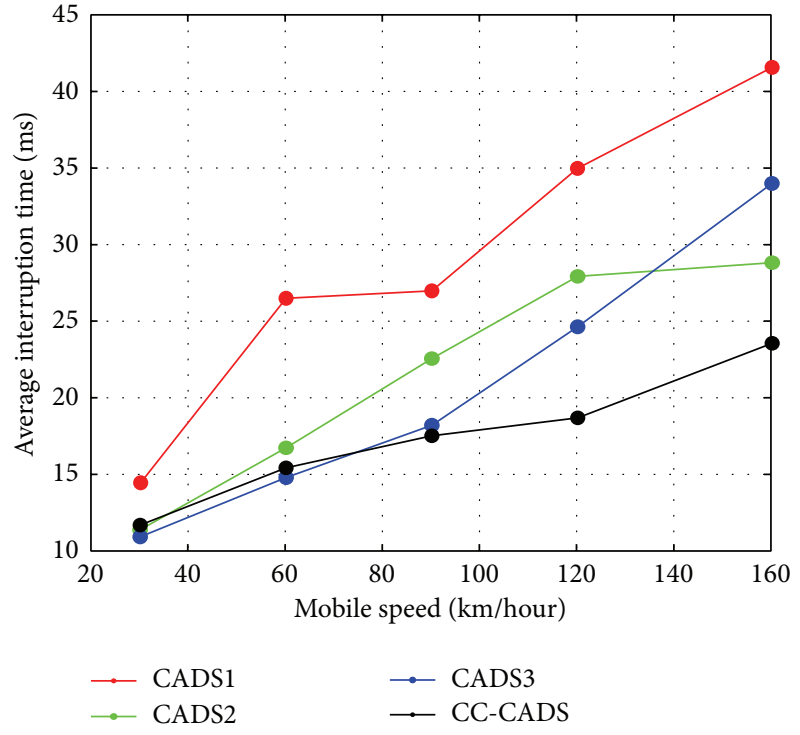

FIGURE 17: User's interruption time based on different CA deployment scenarios.

where $N_{\mathrm{RB}}^{\mathrm{UE}}$ represents the number of PRBs that can be assigned to each $\mathrm{UE}, N_{\mathrm{sc}}^{\mathrm{RB}}$ represents the number of subcarriers per PRB, $N_{\text {symb }}^{\mathrm{sc}}$ represents the number of modulation symbols per subcarrier, and $m_{\text {bit }}^{\text {symb }}$ represents the number of bits per modulation symbol, which depend on the modulation scheme that is adaptively selected. CR is the code rate. 


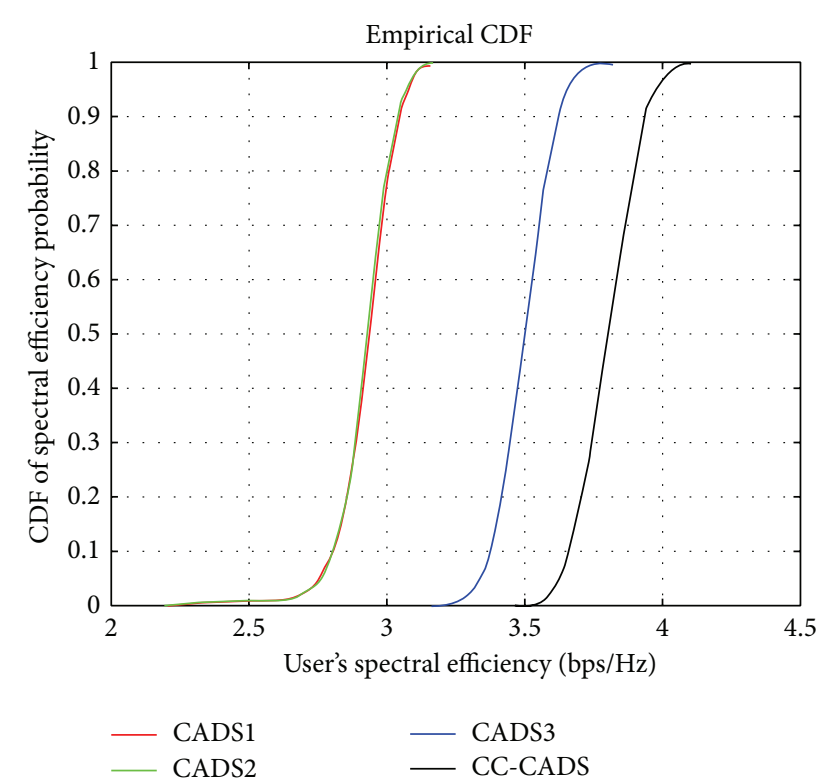

FIGURE 18: User's spectral efficiency based on different CA deployment scenarios.

From (12) to (14), the user's spectral efficiency based on one CC can be formulated by the following formula:

$$
\eta_{j}=\frac{N_{\mathrm{RB}}^{\mathrm{UE}} \times N_{\mathrm{sc}}^{\mathrm{RB}} \times N_{\mathrm{symb}}^{\mathrm{sc}} \times m_{\mathrm{bit}}^{\mathrm{symb}}}{T_{j} \times \omega_{\mathrm{BW}}^{\mathrm{UE}}} \mathrm{CR}_{j}(\mathrm{bps} / \mathrm{Hz}) .
$$

Consequently, the total user's spectral efficiency considering the CA technique based on M CCs can be formulated based on (15), which can be expressed by

$$
\eta_{j}=\sum_{k}^{M} \frac{N_{\mathrm{RB} \_\mathrm{k}}^{\mathrm{UE}} \times N_{\mathrm{sc}}^{\mathrm{RB}} \times N_{\mathrm{symb}}^{\mathrm{sc}} \times m_{\mathrm{bit}}^{\mathrm{symb}}}{T_{j} \times \omega_{\mathrm{BW}}^{\mathrm{UE}}} \mathrm{CR}_{j} .
$$

5.7. Outage Probability. Outage probability $P_{\text {out }}$ is the probability that the instantaneous received SINR $\gamma$ will fall below a given threshold level. The threshold level $\gamma_{\text {Thr }}$ is the minimum SINR level; wireless reception and performance become unacceptable below this level. In mobile cellular systems, the outage probability can be defined mathematically $[19,20]$ and normally represented as follows:

$$
P_{\text {out }}=P\left[\gamma<\gamma_{\mathrm{Thr}}\right]=1-P\left[\gamma>\gamma_{\mathrm{Thr}}\right] .
$$

In our simulation, when the serving SINR for the UE $j$ at position $x$ falls below the threshold, outage probability is recorded. The average outage probability of all users in every simulation cycle is calculated to improve accuracy. Thus, the average outage probability can be simplified from (17) by using the following expression:

$$
P_{\text {out }}(t)=1-\frac{\sum_{j=1}^{N} P\left[\gamma_{j}(t)>\gamma_{\mathrm{Thr}}\right]}{N},
$$

where $N$ is the total number of served users.

\section{Results and Discussion}

In this section, the performance of the proposed CC-CADS is compared with three different conventional CADSs, as presented in Section 2. All the presented results were achieved based on four different CADSs when a hard handover scheme is applied with different mobile speeds. The performances of the conventional and proposed CADSs are presented in terms of serving RSRP, handover, ping-pong, call drop probabilities, interruption time, spectral efficiency, and outage probability.

Figures 12(a) and 12(b) show that the proposed CC-CADS provides a better serving RSRP over the PCC compared with CADS1, CADS2, and CADS3. The presented results represent the average serving RSRP over all UE speeds. The average enhancement gains of serving RSRP by CC-CADS are 3, 3 , and $1.8 \mathrm{~dB}$ over those of CADS1, CADS2, and CADS3, respectively. The CC-CADS provides a stronger serving RSRP than the conventional CADSs.

Figures 13, 14, and 15 show the performed handover, pingpong, and drop calls probabilities based on different CADSs. The presented results represent the average value over all UEs and over all UE's speeds throughout the simulation time. However, Figures 13 and 14 show a noticeable reduction of handover and ping-pong probabilities, respectively, achieved by CC-CCADS compared to CADS1, CADS2, and CADS3. While CADS1 gives the highest handover and ping-pong probabilities compared to the other considered CADSs. The increment of handover and ping-pong probabilities that are resulted by CADS1 due to the utilization of two contagious bands with two antennas directed into the same direction. That leads to results the same path loss over both CCs. Since the fading channel is considered in CADS1, that may lead to providing the same serving RSRP strength over both CCs with small variation between them at any position around the serving eNB. Therefore, that may in turn lead to increasing the probability of PCC handover (PCC HO is the $\mathrm{HO}$ procedure between $\mathrm{CC} 1$ and $\mathrm{CC} 2$ under the same sector to change the serving PCC). Also, it leads to increasing the probability of ping-pong between these two CCs more since path loss differences between both CCs is neglected and fading channel is considered, as shown in Figure 14. In the second scenario (CADS2), utilizing noncontagious bands with two antennas directed in the same direction can reduce the probabilities of the handover and ping-pong, but not so much as shown in Figures 13 and 14, respectively. This reduction is due to the utilization of noncontagious band. In contrast, the overlapping coverages of $\mathrm{CC} 1$ and $\mathrm{CC} 2$ may lead to increasing the oscillation of PCC handover and ping-pong probability, especially at the cell edge of CC2 as shown in Figure 15 at points 1, 2, 3, and 4. Because of that, the reductions of the handover and ping-pong effect appear very little. Also, in the third scenario (CADS3), utilizing noncontagious bands with two antennas directed to two different directions contribute to reducing the probabilities of handover and ping-pong effect less than CADS1 and CADS2, as illustrated in Figures 13 and 14, respectively. However, the presented results indicate that when utilizing contagious bands with two antennas directed to two different directions (CC-CADS) the handover and ping-pong probabilities are 


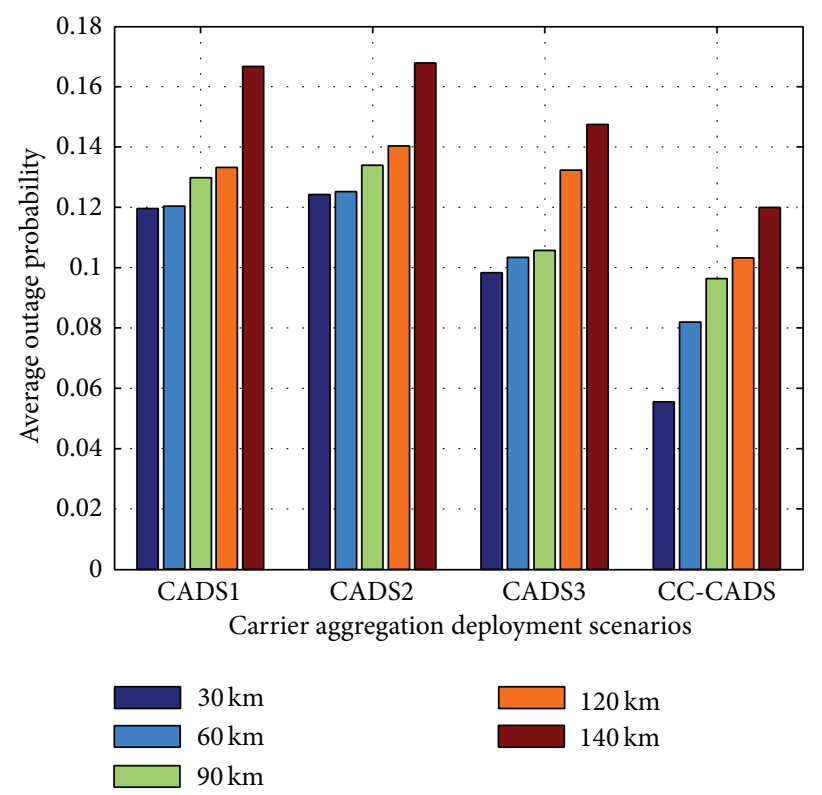

(a) Outage probability with different mobile speed scenarios

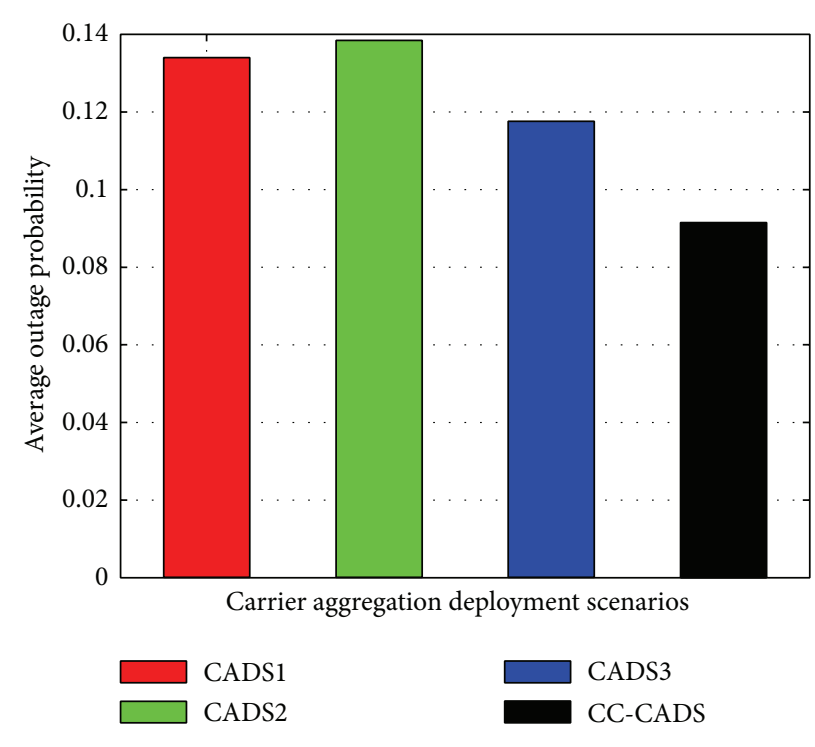

(b) Average user's outage probability

FIGURE 19: Outage probability for different CADSs.

noticeably reduced more compared to CADS1, CADS2, and CADS3. Moreover, CC-CADS gives a noticeable reduction of drop calls, especially when compared to CADS1 and CADS2 as shown in Figure 16. These reductions of the handover, ping-pong, and drop calls probabilities by CC-CADS lead to reducing the interruption time as shown in Figure 17. The average reduction gains of the user's interruption time overall mobile speeds based on CC-CADS are 39.8\%, 19.2\%, and $15.3 \%$ less than those of CADS1, CADS2, and CADS3, respectively.

Figure 18 shows the average spectral efficiency over all UE speeds of CC-CADS and other three different conventional CADSs. The user's spectral efficiency achieved by CC-CADS is significantly better than those achieved by CADS1, CADS2, and CADS3. The average spectral efficiency gains of CCCADS are approximately $30 \%, 30 \%$, and $8.7 \%$ over those of CADS1, CADS2, and CADS3, respectively.

Figures 19(a) and 19(b) show the average outage probability reduction of CC-CADS and other three different conventional CADSs. In Figure 19(a), the outage probability is represented as an average value over all UEs based on different UE speeds scenarios, while in Figure 19(b), outage probability is represented as an average value over all UE's speeds. However, it is clearly shown that the outage probability of CC-CADS is less than that of CADS1, CADS2, and CADS3. The average reduction gains achieved by CC-CADS are approximately $31.7 \%, 33.9 \%$, and $22.2 \%$ less than those of CADS1, CADS2, and CADS3, respectively. Thus, it can be stated that implementing CC-CADS leads to increasing the probability of connection continuity and its stability more than the other CADSs.

Consequently, the performance gains that are achieved by CC-CADS in terms of serving RSRP, handover probability, ping-pong, call drop, interruption time, spectral efficiency, and outage probability reduction can be attributed to the sufficient coverage that is provided by CC-CADS around the serving eNB, whereas the provided sufficient coverage is attributed to the antenna directions and operating frequency, which contribute to system improvement for all locations within the cell.

First, the antenna direction of each CC enhances the overall system performance, where the antenna of each CC is directed to the cell boundary of the other CC. Thus, the main beam direction of $\mathrm{CC} 1$ is directed to the cell boundary of $\mathrm{CC} 2$, whereas the main beam direction of CC2 is directed to the cell boundary of CC1. This scenario increases the beam gains at the cell boundaries, which provides strong serving RSRP to the served UE at cell boundaries, which in turn enhances the serving SINR. Consequently, the spectral efficiency is enhanced, while the handover, ping-pong, drop call, and outage probabilities are decreased; thereby the interruption time is reduced.

Second, the selected carrier frequencies for CC1 and CC2 improve the system performance in the cell. The proposed CC-CADS employs two frequencies located in a contiguous band for $\mathrm{CC} 1$ and CC2. Therefore, the coverage areas provided by these two CCs are almost the same, but each $\mathrm{CC}$ is directed in a different direction. Therefore, sufficient coverage area can be provided in all locations around the serving eNB. The path loss difference between these two CCs is also low because of the close operating bands used for both CCs. Thus, both CCs can support mobility. CC-CADS can provide sufficient coverage area by using both CCs with a negligible difference in the path loss, which enhances the serving RSRP. This condition enhances the serving SINR, which improves spectral efficiency and decreases drop calls, 
outage probability, and interruption time. Thus, it can be stated that CC-CADS supports service continuity better than all conventional CADSs, which in turn leads to increasing connection reliability and its stability through the users mobility.

\section{Conclusion}

Handover performance was investigated in four different carrier aggregation deployment scenarios. The simulation results indicate that the proposed CC-CADS achieved the best system performance evaluation compared with CADS1, CADS2, and CADS3 in terms of serving RSRP, spectral efficiency, interruption time, the performed handover, pingpong, drop calls, and outage probabilities. The enhancement gains achieved by CC-CADS are attributed to the different antenna directions and contiguous operating frequency bands for the aggregated CCs. Therefore, the proposed CCCADS is a suitable CA deployment scenario that can be implemented as a CA deployment scenario option in the LTEAdvanced system.

\section{List of Terminologies Used in the Paper}

CADS: Carrier aggregation deployment scenario

LTE: Long-term evolution

eNB: $\quad$ E-UTRAN node B, also known as evolved node $B$

CC-CADS: Coordinated contiguous-carrier aggregation deployment scenario

CC: $\quad$ Component carrier

3GPP: The 3rd generation partnership project

RSRP: $\quad$ Received signal reference power

Rel. 10: Releases 10 (LTE-Advanced system releases 10)

Rel. 11: Releases 11 (LTE-Advanced system releases 11)

CA: Carrier aggregation

UE: User equipment

CADS1: Carrier aggregation deployment scenario 1

CADS2: Carrier aggregation deployment scenario 2

CADS3: $\quad$ Carrier aggregation deployment scenario 3

CADS4: Carrier aggregation deployment scenario 4

CADS5: Carrier aggregation deployment scenario 5

PCC: $\quad$ Primary component carrier

SCC: $\quad$ Secondary component carrier

RMM: $\quad$ Random mobility model

AMC: $\quad$ Adaptive modulation and coding

RLF: $\quad$ Radio link failure

RRC: $\quad$ Radio resource control

NAS: $\quad$ Nonaccess stratum

PRB: $\quad$ Physical resource block

TTT: $\quad$ Time-to-trigger

HOM: Handover margin

AC: $\quad$ Admission control

DL: Downlink

U-plane: User-plane

HO-Model: Handover-model
SINR: Signal-to-interference-plus-noise-ratio

OFDMA: Orthogonal frequency-division multiple access

HOP: Handover probability

HOPPP: Handover ping-pong probability

DCP: Drop Call probability

DCR: Dropped calls rate

TCR: Total call rate

CR: $\quad$ Code rate.

\section{Conflict of Interests}

The authors declare that there is no conflict of interests regarding the publication of this paper.

\section{Acknowledgment}

The authors would like to acknowledge the financial contribution from Grant reference nos. 01-01-02-SF0789 (MOSTI) and GUP-2012-036 for the publication of this work.

\section{References}

[1] M. Iwamura, K. Etemad, M. Fong, R. Nory, and R. Love, "Carrier aggregation framework in 3GPP LTE-advanced," IEEE Communications Magazine, vol. 48, no. 8, pp. 60-67, 2010.

[2] 3GPP Team, "Evolved Universal Terrestrial Radio Access (EUTRA); Carrier Aggregation; Base Station (BS) radio transmission and reception (Release 10)," in TR 36.808 V10.1.0, Valbonne, France, 2013, http://www.3gpp.org/.

[3] GPP Team, "Simulation assumptions for mobility performance in carrier aggregation," R4-102114, NTT DoCoMo, Montreal, Canada, http://www.3gpp.org/.

[4] GPP Team, "Carrier Aggregation Deployment Scenarios," R2-102490, Beijing, China, pp. 1-3, April 2010, http://www. 3gpp.org/.

[5] GPP Team, "Initial simulations for mobility in carrier aggregation," in R4-102041 Nokia, pp. 1-9, Nokia Siemens Networks, Montreal, Canada, 2010, http://www.3gpp.org/.

[6] GPP Team, "The Impact of CA on Mobility in LTE-A," R1090816 Huawei, Athens, Greece, 2009, http://www.3gpp.org/.

[7] L. Liu, M. Li, J. Zhou et al., "Component carrier management for carrier aggregation in LTE-advanced system," in Proceedings of the IEEE 73rd Vehicular Technology Conference (VTC '11), pp. 1-6, Budapest, Hungary, May 2011.

[8] X. Cao, D. Xiao, and C. Xiu, "Handover performance of relaxing SCell measurement period in LTE-A with carrier aggregation," in Proceedings of the International Conference on Electric Information and Control Engineering (ICEICE '11), pp. 438-441, April 2011.

[9] M. Li, L. Liu, X. She, and L. Chen, "Handover methods in carrier aggregation environment," in Proceedings of the IET International Conference on Communication Technology and Application (ICCTA '11), pp. 213-217, Beijing, China, October 2011.

[10] 3GPP Team, "Evolved Universal Terrestrial Radio Access; Radio Frequency (RF) system scenarios (Release 11)," TR 36.942 V11.0.0., Franch, 2012, http://www.3gpp.org/. 
[11] M. Li, L. Liu, X. She, and L. Chen, "Handover methods considering channel conditions of multiple aggregated carriers," in Proceedings of the IEEE 75th Vehicular Technology Conference (VTC Spring), pp. 1-5, May 2012.

[12] E. Dahlman, S. Parkvall, and J. Sköld, 4G LTE/LTE-Advanced for Mobile Broadband, Ebook, Academic Press, 1st edition, 2011.

[13] GPP Team, "Evolved universal terrestrial radio access (EUTRA); physical channels and modulation (release 11)," in TS 36.211 V11.5.0. France, pp. 1-120, 2013, http://www.3gpp.org/.

[14] 3GPP Team, "Evolved Universal Terrestrial Radio Access (EUTRA); LTE physical layer; General description (Release 11)," TS 36.201 V11.1.0, France, 2012, http://www.3gpp.org.

[15] 3GPP Team, "Evolved Universal Terrestrial Radio Access; Overall description (Release 11)," in TS 36.300 V11.9.0., France, 2014, http://www.3gpp.org/.

[16] P. J. Song and J. Shin, "Method for handover in multi-carrier system," US 2011/0070880 Al, 2011.

[17] L. Zhang, K. Zheng, W. Wang, and L. Huang, "Performance analysis on carrier scheduling schemes in the long-term evolution-advanced system with carrier aggregation," IET Communications, vol. 5, no. 5, pp. 612-619, 2011.

[18] I. Shayea, M. Ismail, and R. Nordin, "Downlink spectral efficiency evaluation with carrier aggregation in LTE-Advanced system employing Adaptive Modulation and Coding schemes," in Proceedings of the IEEE Malaysia International Conference on Communications (MICC '13), pp. 98-103, 2013.

[19] V. Garcia, N. Lebedev, and J. M. Gorce, "Capacity outage probability for multi-cell processing under rayleigh fading," IEEE Communications Letters, vol. 15, no. 8, pp. 801-803, 2011.

[20] J. Paris and D. Morales-Jiménez, “Outage probability analysis for Nakagami-q (Hoyt) fading channels under rayleigh interference," IEEE Transactions on Wireless Communications, vol. 9, no. 4, pp. 1272-1276, 2010. 

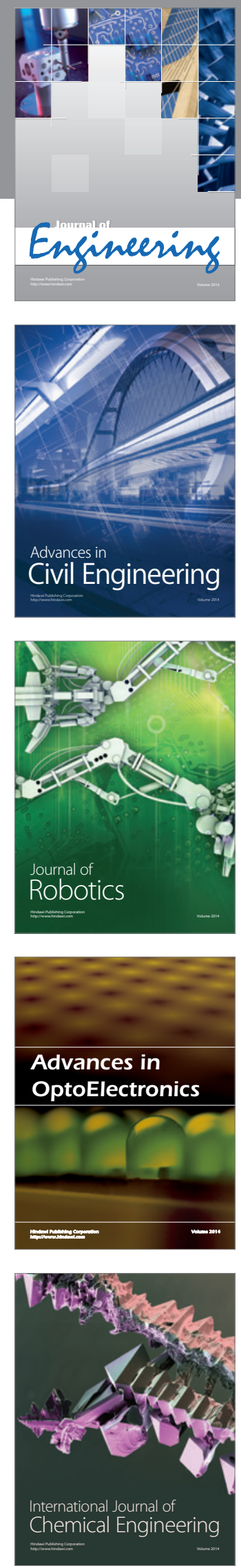

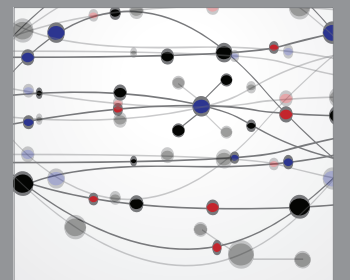

The Scientific World Journal
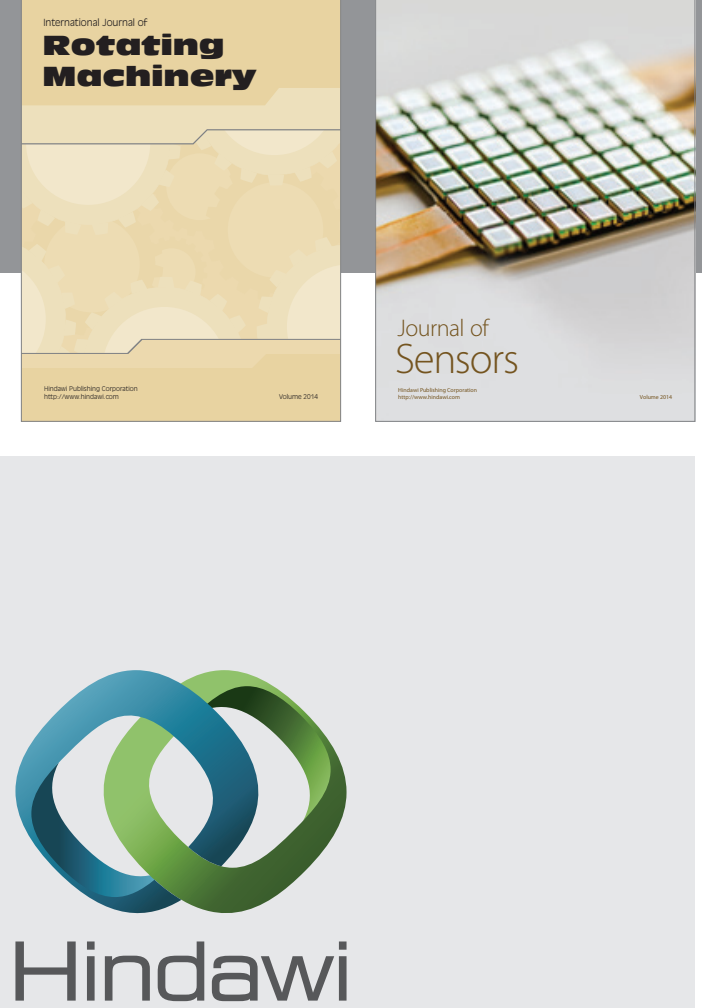

Submit your manuscripts at http://www.hindawi.com
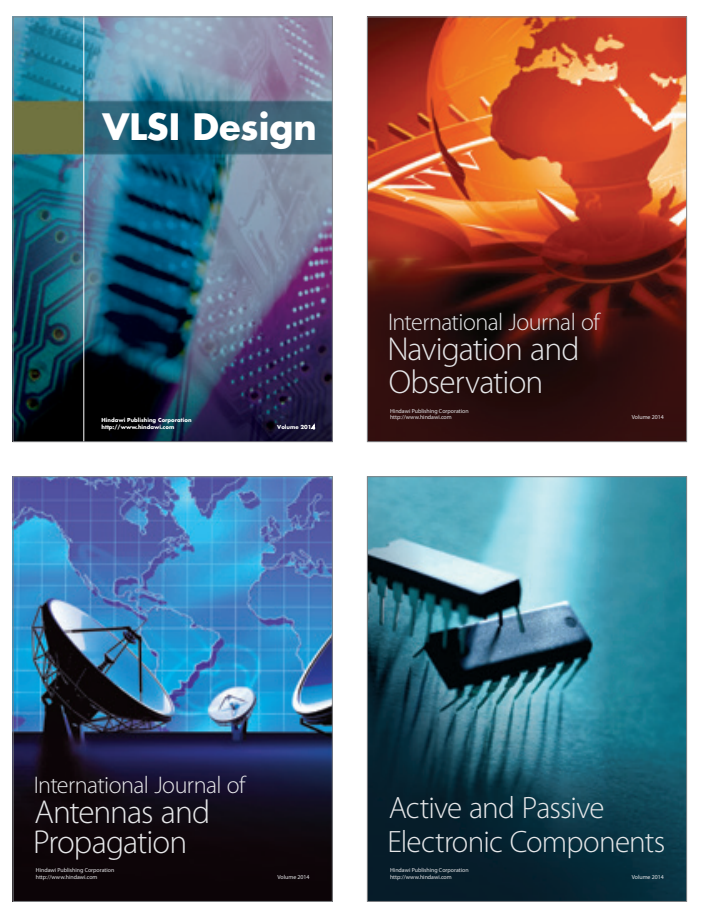
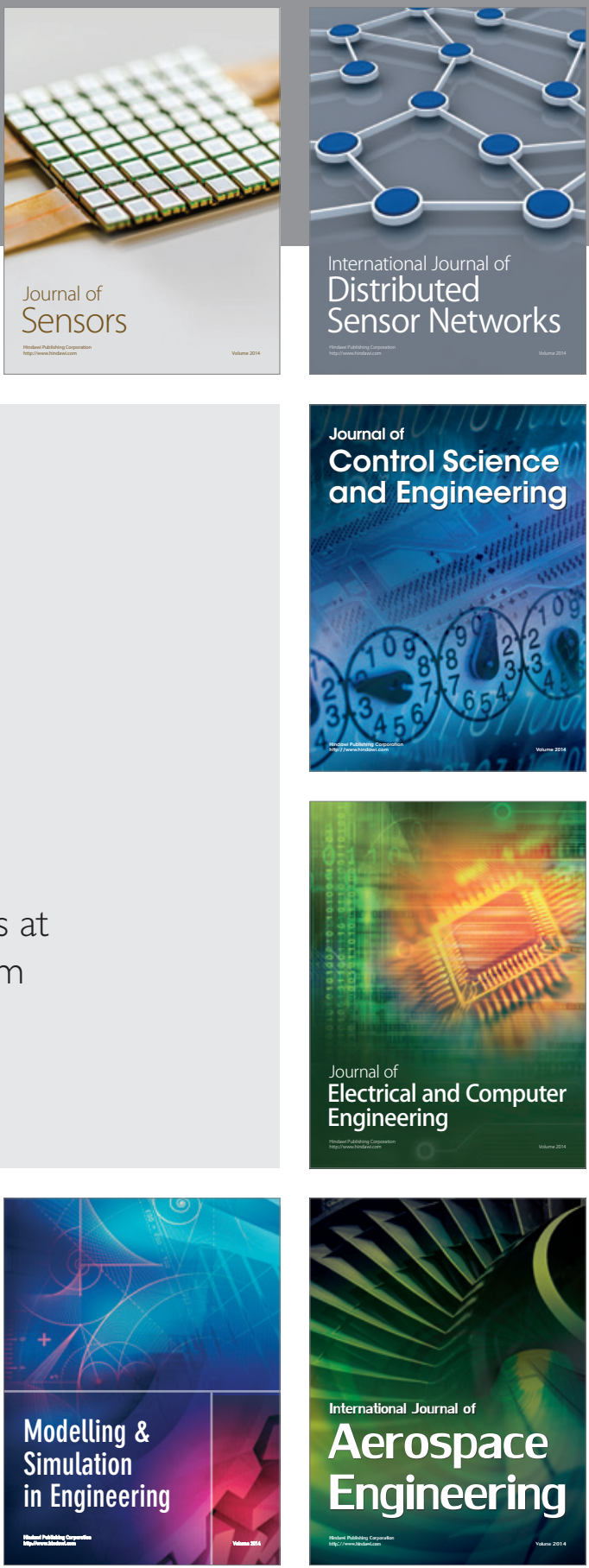

Journal of

Control Science

and Engineering
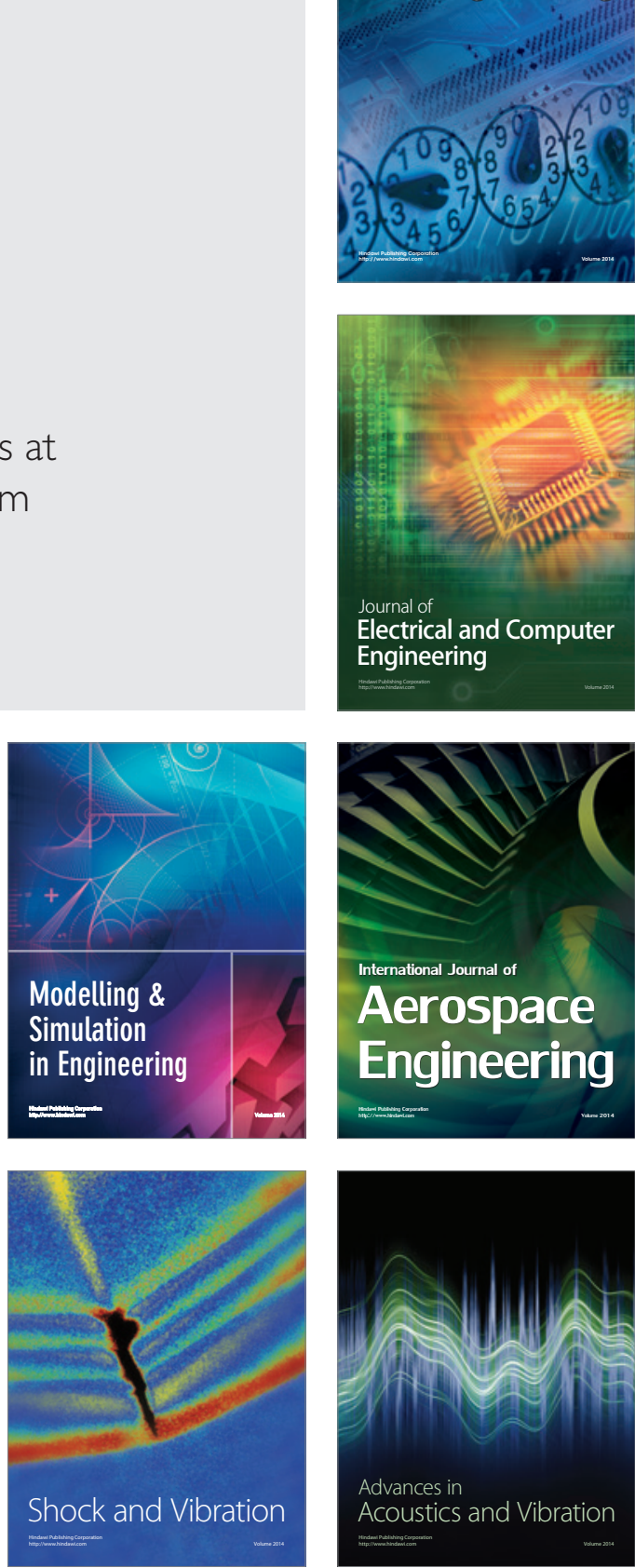